\title{
Dynamical symmetry enhancement near IIA horizons
}

\section{U. Gran, ${ }^{a}$ J. Gutowski, ${ }^{b}$ U. Kayani ${ }^{c}$ and G. Papadopoulos ${ }^{c}$}

${ }^{a}$ Fundamental Physics, Chalmers University of Technology, SE-412 96 Göteborg, Sweden

${ }^{b}$ Department of Mathematics, University of Surrey, Guildford, GU2 7XH, U.K.

${ }^{c}$ Department of Mathematics, King's College London, Strand, London WC2R 2LS, U.K.

E-mail: ulf.gran@chalmers.se, j.gutowski@surrey.ac.uk, usman.kayani@kcl.ac.uk, george.papadopoulos@kcl.ac.uk

ABSTRACT: We show that smooth type IIA Killing horizons with compact spatial sections preserve an even number of supersymmetries, and that the symmetry algebra of horizons with non-trivial fluxes includes an $\mathfrak{s l}(2, \mathbb{R})$ subalgebra. This confirms the conjecture of [1] for type IIA horizons. As an intermediate step in the proof, we also demonstrate new Lichnerowicz type theorems for spin bundle connections whose holonomy is contained in a general linear group.

KEYwords: Black Holes in String Theory, Supergravity Models

ARXIV EPRINT: 1409.6303 


\section{Contents}

1 Introduction $\quad 2$

2 Horizon fields and KSEs $\quad 4$

2.1 IIA fields and field equations 4

2.2 Horizon fields, Bianchi identities and field equations 5

$\begin{array}{lll}2.3 & \text { Integration of KSEs along the lightcone } & 7\end{array}$

2.4 Independent KSEs 8

3 Supersymmetry enhancement $\quad 9$

3.1 Horizon Dirac equations 9

3.2 A Lichnerowicz type theorem for $\mathcal{D}^{(+)} \quad 9$

3.3 A Lichnerowicz type theorem for $\mathcal{D}^{(-)} \quad 10$

3.4 Supersymmetry enhancement 11

4 Construction of $\eta_{+}$from $\eta_{-}$Killing spinors $\quad 11$

$5 \quad$ The $\mathfrak{s l}(2, \mathbb{R})$ symmetry of IIA horizons $\quad \mathbf{1 3}$

$\begin{array}{ll}5.1 \text { Killing vectors } & 13\end{array}$

$\begin{array}{lll}5.2 & \text { The geometry of } \mathcal{S} & 14\end{array}$

$\begin{array}{ll}5.3 \mathfrak{s l}(2, \mathbb{R}) \text { symmetry of IIA-horizons } & 14\end{array}$

6 Conclusions $\quad 15$

$\begin{array}{ll}\text { A Horizon Bianchi identities and field equations } & 15\end{array}$

B Integrability conditions and KSEs $\quad 16$

$\begin{array}{ll}\text { B.1 Dilatino KSE } & 17\end{array}$

$\begin{array}{ll}\text { B.2 Independent KSEs } & 17\end{array}$

$\begin{array}{lll}\text { B.2.1 The (B.5) condition } & 18\end{array}$

$\begin{array}{ll}\text { B.2.2 The (B.8) condition } & 19\end{array}$

$\begin{array}{ll}\text { B.2.3 The (B.1) condition } & 19\end{array}$

B.2.4 The + (B.7) condition linear in $u \quad 20$

B.2.5 The (B.2) condition 20

$\begin{array}{ll}\text { B.2.6 The (B.3) condition } & 21\end{array}$

$\begin{array}{lll}\text { B.2.7 The }+(\text { B.4) condition linear in } u & 21\end{array}$

C Calculation of Laplacian of $\left\|\eta_{ \pm}\right\|^{2} \quad 21$ 


\section{Introduction}

It has been conjectured in [1], following earlier work in [2] and [3], that

- the number of Killing spinors $N, N \neq 0$, of Killing horizons in supergravity is given by

$$
N=2 N_{-}+\operatorname{Index}\left(D_{E}\right)
$$

where $N_{-} \in \mathbb{N}_{>0}$ and $D_{E}$ is a Dirac operator twisted by a vector bundle $E$, defined on the spatial horizon section $\mathcal{S}$, which depends on the gauge symmetries of the supergravity theory in question, and

- that horizons with non-trivial fluxes and $N_{-} \neq 0$ admit an $\mathfrak{s l}(2, \mathbb{R})$ symmetry subalgebra.

This conjecture encompasses the essential features of (super)symmetry enhancement near black hole Killing horizons, and some features of the same phenomenon near brane horizons, previously obtained in the literature based on a case-by-case investigation [46]. Symmetry enhancement near black hole and brane horizons has been instrumental in the development of the AdS/CFT correspondence [7]. So far, this conjecture has been established for minimal 5-dimensional gauged supergravity, $\mathrm{D}=11$ M-theory, and $\mathrm{D}=10$ IIB supergravity [1-3].

The main purpose of this paper is to prove the above conjecture for Killing horizons in IIA supergravity. The proof is based on three assumptions. First, it is assumed that the Killing horizons admit at least one supersymmetry, second that the near horizon geometries are smooth and third that the spatial horizon sections are compact without boundary ${ }^{1}$. It turns out that for IIA horizons, the contribution from the index of $D_{E}$ in the expression for $N$ in (1.1) vanishes and therefore one concludes that IIA horizons always preserve an even number of supersymmetries, i.e.

$$
N=2 N_{-} .
$$

Furthermore from the second part of the conjecture, one concludes that all supersymmetric IIA horizons with non-trivial fluxes admit an $\mathfrak{s l}(2, \mathbb{R})$ symmetry subalgebra.

To prove the conjecture, we first adapt the description of black hole near horizon geometries of $[8,9]$ to IIA supergravity. The metric and the remaining fields of IIA horizons are given in (2.9). We then decompose the Killing spinor as $\epsilon=\epsilon_{+}+\epsilon_{-}$using the lightcone projectors $\Gamma_{ \pm} \epsilon_{ \pm}=0$ and integrate the Killing spinor equations (KSEs) of IIA supergravity along the two lightcone directions. These directions arise naturally in the description of near horizon geometries. As a result, the Killing spinors of IIA horizons can be written as $\epsilon=\epsilon\left(u, r, \eta_{ \pm}\right)$, where the dependence on the coordinates $u, r$ is explicit and $\eta_{ \pm}$are spinors

\footnotetext{
${ }^{1}$ This is not an essential assumption and it may be weakened. However to extend our proof to horizons with non-compact $\mathcal{S}$, one has to impose appropriate boundary conditions on the fields. Because of this, and for simplicity, we shall not do this here and throughout this paper we shall assume that $\mathcal{S}$ is compact without boundary.
} 
which depend only on the coordinates of the spatial horizon section $\mathcal{S}$ given by the equation $u=r=0$.

As a key next step in the proof, we demonstrate that the remaining independent KSEs are those obtained from the KSEs of IIA supergravity after naively restricting them to $\mathcal{S}$. In particular, we find after an extensive use of the field equations and Bianchi identities that all the integrability conditions that arise along the lightcone directions, and the mixed directions between the lightcone and the $\mathcal{S}$ directions, are automatically satisfied. The independent KSEs on $\mathcal{S}$ split into two sets $\left\{\nabla^{( \pm)}, \mathcal{A}^{( \pm)}\right\}$of two KSEs with each set acting on the spinors $\eta_{ \pm}$distinguished by the choice of lightcone direction, where $\nabla^{( \pm)}$ are derived from the gravitino KSE of IIA supergravity and $\mathcal{A}^{( \pm)}$are associated to the dilatino KSE of IIA supergravity. In addition we demonstrate that if $\eta_{-}$is a Killing spinor on $\mathcal{S}$, then $\eta_{+}=\Gamma_{+} \Theta_{-} \eta_{-}$also solves the KSEs, where $\Theta_{-}$depends on the fluxes and the spacetime metric.

To show that the number of Killing spinors of IIA horizons is even, it suffices to show that there are as many $\eta_{+}$Killing spinors as $\eta_{-}$Killing spinors. For this, we first identify the Killing spinors $\eta_{ \pm}$with the zero modes of Dirac-like operators $\mathscr{D}^{( \pm)}$coupled to fluxes. These are defined as $\mathscr{D}^{( \pm)}=\mathcal{D}^{( \pm)}+q \mathcal{A}^{( \pm)}$, where $\mathcal{D}^{( \pm)}$is the Dirac operator constructed from $\nabla^{( \pm)}$. It is then shown that for a suitable choice of $q$ all zero modes of these Dirac-like operators are in 1-1 correspondence with the Killing spinors.

The proof of the above correspondence between zero modes and Killing spinors for the $\mathscr{D}^{(+)}$operator utilizes the Hopf maximum principle and relies on the formula (3.6). Incidentally, this also establishes that $\left\|\eta_{+}\right\|$is constant. The proof for the $\mathscr{D}^{(-)}$operator uses the partial integration of the formula (3.9) and this is similar to the classical Lichnerowicz theorem for the Dirac operator. In both cases, the proofs rely on the smoothness of data and the assumption that $\mathcal{S}$ is compact without boundary.

Therefore, the number of Killing spinors of IIA horizons is $N=N_{+}+N_{-}$, where $N_{ \pm}$ are the dimensions of the kernels of the $\mathscr{D}^{( \pm)}$operators. On the other hand, one can show that the zero modes of $\mathscr{D}^{(-)}$are in 1-1 correspondence with the zero modes of the adjoint $\left(\mathscr{D}^{(+)}\right)^{\dagger}$ of $\mathscr{D}^{(+)}$. As a result $N_{+}-N_{-}$is the index of $\mathscr{D}^{(+)}$. This vanishes as it is equal to the index of the Dirac operator acting on the spinor bundle constructed from the $\mathbf{1 6}$ dimensional Majorana representation of $\operatorname{Spin}(8)$. As a result $N_{+}=N_{-}$and the number of supersymmetries preserved by IIA horizons is even, which proves the first part of the conjecture.

To prove that IIA horizons admit an $\mathfrak{s l}(2, \mathbb{R})$ symmetry subalgebra, we use the fact that if $\eta_{-}$is a Killing spinor then $\eta_{+}=\Gamma_{+} \Theta_{-} \eta_{-}$is also a Killing spinor. To see this we demonstrate that if the fluxes do not vanish, the kernel of $\Theta_{-}$is $\{0\}$, and so $\eta_{+} \neq 0$. Using the Killing spinors now constructed from $\eta_{-}$and $\eta_{+}=\Gamma_{+} \Theta_{-} \eta_{-}$, we prove that the spacetime admits three Killing vectors, which leave all the fields invariant, satisfying an $\mathfrak{s l}(2, \mathbb{R})$ algebra. This completes the proof of the conjecture for IIA horizons.

The results presented above for horizons in IIA supergravity are not a straightforward consequence of those we have obtained for M-horizons in [3]. Although IIA supergravity is the dimensional reduction of 11-dimensional supergravity, it is well known that, after the truncation of Kaluza-Klein modes, not all of the supersymmetry of 11-dimensional 
solutions survives the reduction to IIA; for a detailed analysis of these issues see $[10,11]$. As a result, for example, it does not follow automatically that IIA horizons preserve an even number of supersymmetries because M-horizons do, as shown in [3]. For this, one has to demonstrate that the Killing spinors of 11-dimensional horizons are always annihilated in pairs upon the action of the spinorial Lie derivative along any space-like vector field $X$ which leaves the fields invariant and has closed orbits. However since we have shown that both IIA and M-theory horizons preserve an even number of supersymmetries, one concludes that if the reduction process breaks some supersymmetry, then it always breaks an even number of supersymmetries. Moreover, our IIA analysis presented here has several advantages. In particular, it explains why the index contribution in (1.1) vanishes based on the analysis of section 3 and also provides an explicit expression for the generators of the $\mathfrak{s l}(2, \mathbb{R})$ symmetry in section 5 . Both these results are not directly accessible from an 11-dimensional analysis. Furthermore, the proof of the IIA Lichnerowicz type theorems is more general than that presented for 11-dimensional horizons in [3] because of the presence of additional parameters like $q$ and $\kappa$, ie the IIA Lichnerowicz type theorems are valid for a more general class of operators than those that one constructs from the dimensional reduction of those of [3]. In addition, the identification of the independent KSEs for IIA horizons in section 2.4 will be useful in a future investigation of the geometry of IIA horizons.

This paper is organized as follows. In section 2, we identify the independent KSEs for IIA horizons. In section 3 , we establish the equivalence between zero modes of $\mathscr{D}^{( \pm)}$and Killing spinors, and show that the number of supersymmetries preserved by IIA horizons is even. In section 4 , we show that $\eta_{+}=\Gamma_{+} \Theta_{-} \eta_{-} \neq 0$. In section 5 , we prove that IIA horizons with non-trivial fluxes admit an $\mathfrak{s l}(2, \mathbb{R})$ symmetry subalgebra and in section 6 we give our conclusions. In appendix A, we give a list of Bianchi identities and field equations that are implied by the (independent) ones listed in section 2. In appendix B, we identify the independent KSEs, and in appendix $\mathrm{C}$ we establish the formulae (3.6) and (3.9).

\section{Horizon fields and KSEs}

\subsection{IIA fields and field equations}

The bosonic field content of IIA supergravity [12-15] are the spacetime metric $g$, the dilaton $\Phi$, the 2-form NS-NS gauge potential $B$, and the 1 -form and the 3 -form RR gauge potentials $A$ and $C$, respectively. In addition, the theory has non-chiral fermionic fields consisting of a Majorana gravitino and a Majorana dilatino but these are set to zero in all the computations that follow. The bosonic field strengths of IIA supergravity in the conventions of [16] are

$$
F=d A, \quad H=d B, \quad G=d C-H \wedge A .
$$

These lead to the Bianchi identities

$$
d F=0, \quad d H=0, \quad d G=F \wedge H .
$$


The bosonic part of the IIA action in the string frame is

$$
\begin{aligned}
S= & \int \sqrt{-g}\left(e^{-2 \Phi}\left(R+4 \nabla_{\mu} \Phi \nabla^{\mu} \Phi-\frac{1}{12} H_{\lambda_{1} \lambda_{2} \lambda_{3}} H^{\lambda_{1} \lambda_{2} \lambda_{3}}\right)\right. \\
& \left.-\frac{1}{4} F_{\mu \nu} F^{\mu \nu}-\frac{1}{48} G_{\mu_{1} \mu_{2} \mu_{3} \mu_{4}} G^{\mu_{1} \mu_{2} \mu_{3} \mu_{4}}\right)+\frac{1}{2} d C \wedge d C \wedge B .
\end{aligned}
$$

This leads to the Einstein equation

$$
\begin{aligned}
R_{\mu \nu}= & -2 \nabla_{\mu} \nabla_{\nu} \Phi+\frac{1}{4} H_{\mu \lambda_{1} \lambda_{2}} H_{\nu}^{\lambda_{1} \lambda_{2}}+\frac{1}{2} e^{2 \Phi} F_{\mu \lambda} F_{\nu}{ }^{\lambda}+\frac{1}{12} e^{2 \Phi} G_{\mu \lambda_{1} \lambda_{2} \lambda_{3}} G_{\nu}{ }^{\lambda_{1} \lambda_{2} \lambda_{3}} \\
& +g_{\mu \nu}\left(-\frac{1}{8} e^{2 \Phi} F_{\lambda_{1} \lambda_{2}} F^{\lambda_{1} \lambda_{2}}-\frac{1}{96} e^{2 \Phi} G_{\lambda_{1} \lambda_{2} \lambda_{3} \lambda_{4}} G^{\lambda_{1} \lambda_{2} \lambda_{3} \lambda_{4}}\right),
\end{aligned}
$$

the dilaton field equation

$$
\begin{aligned}
\nabla^{\mu} \nabla_{\mu} \Phi= & 2 \nabla_{\lambda} \Phi \nabla^{\lambda} \Phi-\frac{1}{12} H_{\lambda_{1} \lambda_{2} \lambda_{3}} H^{\lambda_{1} \lambda_{2} \lambda_{3}}+\frac{3}{8} e^{2 \Phi} F_{\lambda_{1} \lambda_{2}} F^{\lambda_{1} \lambda_{2}} \\
& +\frac{1}{96} e^{2 \Phi} G_{\lambda_{1} \lambda_{2} \lambda_{3} \lambda_{4}} G^{\lambda_{1} \lambda_{2} \lambda_{3} \lambda_{4}}
\end{aligned}
$$

the 2-form field equation

$$
\nabla^{\mu} F_{\mu \nu}+\frac{1}{6} H^{\lambda_{1} \lambda_{2} \lambda_{3}} G_{\lambda_{1} \lambda_{2} \lambda_{3} \nu}=0
$$

the 3 -form field equation

$$
\nabla_{\lambda}\left(e^{-2 \Phi} H^{\lambda \mu \nu}\right)-\frac{1}{2} G^{\mu \nu \lambda_{1} \lambda_{2}} F_{\lambda_{1} \lambda_{2}}+\frac{1}{1152} \epsilon^{\mu \nu \lambda_{1} \lambda_{2} \lambda_{3} \lambda_{4} \lambda_{5} \lambda_{6} \lambda_{7} \lambda_{8}} G_{\lambda_{1} \lambda_{2} \lambda_{3} \lambda_{4}} G_{\lambda_{5} \lambda_{6} \lambda_{7} \lambda_{8}}=0,
$$

and the 4-form field equation

$$
\nabla_{\mu} G^{\mu \nu_{1} \nu_{2} \nu_{3}}+\frac{1}{144} \epsilon^{\nu_{1} \nu_{2} \nu_{3} \lambda_{1} \lambda_{2} \lambda_{3} \lambda_{4} \lambda_{5} \lambda_{6} \lambda_{7}} G_{\lambda_{1} \lambda_{2} \lambda_{3} \lambda_{4}} H_{\lambda_{5} \lambda_{6} \lambda_{7}}=0 .
$$

This completes the description of the dynamics of the bosonic part of IIA supergravity.

\subsection{Horizon fields, Bianchi identities and field equations}

The description of the metric near extreme Killing horizons as expressed in Gaussian null coordinates $[8,9]$ can be adapted to include all IIA fields. In particular, one writes

$$
\begin{aligned}
d s^{2} & =2 \mathbf{e}^{+} \mathbf{e}^{-}+\delta_{i j} \mathbf{e}^{i} \mathbf{e}^{j}, & & G=\mathbf{e}^{+} \wedge \mathbf{e}^{-} \wedge X+r \mathbf{e}^{+} \wedge Y+\tilde{G}, \\
H & =\mathbf{e}^{+} \wedge \mathbf{e}^{-} \wedge L+r \mathbf{e}^{+} \wedge M+\tilde{H}, & & F=\mathbf{e}^{+} \wedge \mathbf{e}^{-} S+r \mathbf{e}^{+} \wedge T+\tilde{F},
\end{aligned}
$$

where we have introduced the frame

$$
\mathbf{e}^{+}=d u, \quad \mathbf{e}^{-}=d r+r h-\frac{1}{2} r^{2} \Delta d u, \quad \mathbf{e}^{i}=e_{I}^{i} d y^{I},
$$

and the dependence on the coordinates $u$ and $r$ is explicitly given. Moreover $\Phi$ and $\Delta$ are 0 -forms, $h, L$ and $T$ are 1-forms, $X, M$ and $\tilde{F}$ are 2-forms, $Y, \tilde{H}$ are 3 -forms and $\tilde{G}$ is 
a 4 -form on the spatial horizon section $\mathcal{S}$, which is the co-dimension 2 submanifold given by the equation $r=u=0$, i.e. all these components of the fields depend only on the coordinates of $\mathcal{S}$. It should be noted that one of our assumptions is that all these forms on $\mathcal{S}$ are sufficiently differentiable, i.e. we require at least $C^{2}$ differentiability so that all the field equations and Bianchi identities are valid.

Substituting the fields (2.9) into the Bianchi identities of IIA supergravity, one finds that

$$
\begin{aligned}
& M=d_{h} L, \quad T=d_{h} S, \quad Y=d_{h} X-L \wedge \tilde{F}-S \tilde{H}, \\
& d \tilde{G}=\tilde{H} \wedge \tilde{F}, \quad d \tilde{H}=d \tilde{F}=0,
\end{aligned}
$$

where $d_{h} \theta \equiv d \theta-h \wedge \theta$ for any form $\theta$. These are the only independent Bianchi identities, see appendix A.

Similarly, substituting the horizon fields into the field equations of IIA supergravity, we find that the 2 -form field equation (2.6) gives

$$
\tilde{\nabla}^{i} \tilde{F}_{i k}-h^{i} \tilde{F}_{i k}+T_{k}-L^{i} X_{i k}+\frac{1}{6} \tilde{H}^{\ell_{1} \ell_{2} \ell_{3}} \tilde{G}_{\ell_{1} \ell_{2} \ell_{3} k}=0,
$$

the 3-form field equation (2.7) gives

$$
\tilde{\nabla}^{i}\left(e^{-2 \Phi} L_{i}\right)-\frac{1}{2} \tilde{F}^{i j} X_{i j}+\frac{1}{1152} \epsilon^{\ell_{1} \ell_{2} \ell_{3} \ell_{4} \ell_{5} \ell_{6} \ell_{7} \ell_{8}} \tilde{G}_{\ell_{1} \ell_{2} \ell_{3} \ell_{4}} \tilde{G}_{\ell_{5} \ell_{6} \ell_{7} \ell_{8}}=0
$$

and

$$
\begin{aligned}
\tilde{\nabla}^{i}\left(e^{-2 \Phi} \tilde{H}_{i m n}\right)-e^{-2 \Phi} h^{i} \tilde{H}_{i m n}+ & e^{-2 \Phi} M_{m n}+S X_{m n}-\frac{1}{2} \tilde{F}^{i j} \tilde{G}_{i j m n} \\
& -\frac{1}{48} \epsilon_{m n}{ }^{\ell_{1} \ell_{2} \ell_{3} \ell_{4} \ell_{5} \ell_{6}} X_{\ell_{1} \ell_{2}} \tilde{G}_{\ell_{3} \ell_{4} \ell_{5} \ell_{6}}=0,
\end{aligned}
$$

and the 4-form field equation (2.8) gives

$$
\tilde{\nabla}^{i} X_{i k}+\frac{1}{144} \epsilon_{k}^{\ell_{1} \ell_{2} \ell_{3} \ell_{4} \ell_{5} \ell_{6} \ell_{7}} \tilde{G}_{\ell_{1} \ell_{2} \ell_{3} \ell_{4}} \tilde{H}_{\ell_{5} \ell_{6} \ell_{7}}=0
$$

and

$\tilde{\nabla}^{i} \tilde{G}_{i j k q}+Y_{j k q}-h^{i} \tilde{G}_{i j k q}-\frac{1}{12} \epsilon_{j k q}{ }^{\ell_{1} \ell_{2} \ell_{3} \ell_{4} \ell_{5}} X_{\ell_{1} \ell_{2}} \tilde{H}_{\ell_{3} \ell_{4} \ell_{5}}-\frac{1}{24} \epsilon_{j k q}{ }^{\ell_{1} \ell_{2} \ell_{3} \ell_{4} \ell_{5}} \tilde{G}_{\ell_{1} \ell_{2} \ell_{3} \ell_{4}} L_{\ell_{5}}=0$,

where $\tilde{\nabla}$ is the Levi-Civita connection of the metric on $\mathcal{S}$. In addition, the dilaton field equation (2.5) becomes

$$
\begin{aligned}
\tilde{\nabla}^{i} \tilde{\nabla}_{i} \Phi-h^{i} \tilde{\nabla}_{i} \Phi= & 2 \tilde{\nabla}_{i} \Phi \tilde{\nabla}^{i} \Phi+\frac{1}{2} L_{i} L^{i}-\frac{1}{12} \tilde{H}_{\ell_{1} \ell_{2} \ell_{3}} \tilde{H}^{\ell_{1} \ell_{2} \ell_{3}}-\frac{3}{4} e^{2 \Phi} S^{2} \\
& +\frac{3}{8} e^{2 \Phi} \tilde{F}_{i j} \tilde{F}^{i j}-\frac{1}{8} e^{2 \Phi} X_{i j} X^{i j}+\frac{1}{96} e^{2 \Phi} \tilde{G}_{\ell_{1} \ell_{2} \ell_{3} \ell_{4}} \tilde{G}^{\ell_{1} \ell_{2} \ell_{3} \ell_{4}} .
\end{aligned}
$$

It remains to evaluate the Einstein field equation. This gives

$$
\begin{aligned}
\frac{1}{2} \tilde{\nabla}^{i} h_{i}-\Delta-\frac{1}{2} h^{2}= & h^{i} \tilde{\nabla}_{i} \Phi-\frac{1}{2} L_{i} L^{i}-\frac{1}{4} e^{2 \Phi} S^{2}-\frac{1}{8} e^{2 \Phi} X_{i j} X^{i j} \\
& -\frac{1}{8} e^{2 \Phi} \tilde{F}_{i j} \tilde{F}^{i j}-\frac{1}{96} e^{2 \Phi} \tilde{G}_{\ell_{1} \ell_{2} \ell_{3} \ell_{4}} \tilde{G}^{\ell_{1} \ell_{2} \ell_{3} \ell_{4}},
\end{aligned}
$$


and

$$
\begin{aligned}
\tilde{R}_{i j}= & -\tilde{\nabla}_{(i} h_{j)}+\frac{1}{2} h_{i} h_{j}-2 \tilde{\nabla}_{i} \tilde{\nabla}_{j} \Phi-\frac{1}{2} L_{i} L_{j}+\frac{1}{4} \tilde{H}_{i \ell_{1} \ell_{2}} \tilde{H}_{j}^{\ell_{1} \ell_{2}} \\
& +\frac{1}{2} e^{2 \Phi} \tilde{F}_{i \ell} \tilde{F}_{j}^{\ell}-\frac{1}{2} e^{2 \Phi} X_{i \ell} X_{j}^{\ell}+\frac{1}{12} e^{2 \Phi} \tilde{G}_{i \ell_{1} \ell_{2} \ell_{3}} \tilde{G}_{j}^{\ell_{1} \ell_{2} \ell_{3}} \\
& +\delta_{i j}\left(\frac{1}{4} e^{2 \Phi} S^{2}-\frac{1}{8} e^{2 \Phi} \tilde{F}_{\ell_{1} \ell_{2}} \tilde{F}^{\ell_{1} \ell_{2}}+\frac{1}{8} e^{2 \Phi} X_{\ell_{1} \ell_{2}} X^{\ell_{1} \ell_{2}}-\frac{1}{96} e^{2 \Phi} \tilde{G}_{\ell_{1} \ell_{2} \ell_{3} \ell_{4}} \tilde{G}^{\ell_{1} \ell_{2} \ell_{3} \ell_{4}}\right) .
\end{aligned}
$$

Above we have only stated the independent field equations. In fact, after substituting the near horizon geometries into the IIA field equations, there are additional equations that arise. However, these are all implied from the above field equations and Bianchi identities. For completeness, these additional equations are given in appendix A.

To summarize, the independent Bianchi identities and field equations are given in (2.11)$(2.19)$.

\subsection{Integration of KSEs along the lightcone}

The KSEs of IIA supergravity are the vanishing conditions of the gravitino and dilatino supersymmetry variations evaluated at the locus where all fermions vanish. These can be expressed as

$$
\begin{aligned}
\mathcal{D}_{\mu} \epsilon \equiv & \nabla_{\mu} \epsilon+\frac{1}{8} H_{\mu \nu_{1} \nu_{2}} \Gamma^{\nu_{1} \nu_{2}} \Gamma_{11} \epsilon+\frac{1}{16} e^{\Phi} F_{\nu_{1} \nu_{2}} \Gamma^{\nu_{1} \nu_{2}} \Gamma_{\mu} \Gamma_{11} \epsilon \\
& +\frac{1}{8 \cdot 4 !} e^{\Phi} G_{\nu_{1} \nu_{2} \nu_{3} \nu_{4}} \Gamma^{\nu_{1} \nu_{2} \nu_{3} \nu_{4}} \Gamma_{\mu} \epsilon=0, \\
\mathcal{A} \epsilon \equiv & \partial_{\mu} \Phi \Gamma^{\mu} \epsilon+\frac{1}{12} H_{\mu_{1} \mu_{2} \mu_{3}} \Gamma^{\mu_{1} \mu_{2} \mu_{3}} \Gamma_{11} \epsilon+\frac{3}{8} e^{\Phi} F_{\mu_{1} \mu_{2}} \Gamma^{\mu_{1} \mu_{2}} \Gamma_{11} \epsilon \\
& +\frac{1}{4 \cdot 4 !} e^{\Phi} G_{\mu_{1} \mu_{2} \mu_{3} \mu_{4}} \Gamma^{\mu_{1} \mu_{2} \mu_{3} \mu_{4}} \epsilon=0,
\end{aligned}
$$

where $\epsilon$ is the supersymmetry parameter which from now on is taken to be a Majorana, but not Weyl, commuting spinor of $\operatorname{Spin}(9,1)$. In what follows, we shall refer to the $\mathcal{D}$ operator as the supercovariant connection.

Supersymmetric IIA horizons are those for which there exists an $\epsilon \neq 0$ that is a solution of the KSEs. To find the conditions on the fields required for such a solution to exist, we first integrate along the two lightcone directions, i.e. we integrate the KSEs along the $u$ and $r$ coordinates. To do this, we decompose $\epsilon$ as

$$
\epsilon=\epsilon_{+}+\epsilon_{-},
$$

where $\Gamma_{ \pm} \epsilon_{ \pm}=0$, and find that

$$
\epsilon_{+}=\phi_{+}(u, y), \quad \epsilon_{-}=\phi_{-}+r \Gamma_{-} \Theta_{+} \phi_{+},
$$

and

$$
\phi_{-}=\eta_{-}, \quad \phi_{+}=\eta_{+}+u \Gamma_{+} \Theta_{-} \eta_{-},
$$


where

$\Theta_{ \pm}=\frac{1}{4} h_{i} \Gamma^{i} \mp \frac{1}{4} \Gamma_{11} L_{i} \Gamma^{i}-\frac{1}{16} e^{\Phi} \Gamma_{11}\left( \pm 2 S+\tilde{F}_{i j} \Gamma^{i j}\right)-\frac{1}{8 \cdot 4 !} e^{\Phi}\left( \pm 12 X_{i j} \Gamma^{i j}+\tilde{G}_{i j k l} \Gamma^{i j k l}\right)$,

and $\eta_{ \pm}$depend only on the coordinates of the spatial horizon section $\mathcal{S}$. As spinors on $\mathcal{S}$, $\eta_{ \pm}$are sections of the $\operatorname{Spin}(8)$ bundle on $\mathcal{S}$ associated with the Majorana representation. Equivalently, the $\operatorname{Spin}(9,1)$ bundle $S$ on the spacetime when restricted to $\mathcal{S}$ decomposes as $S=S_{-} \oplus S_{+}$according to the lightcone projections $\Gamma_{ \pm}$. Although $S_{ \pm}$are distinguished by the lightcone chirality, they are isomorphic as $\operatorname{Spin}(8)$ bundles over $\mathcal{S}$. We shall use this in the counting of supersymmetries of IIA horizons.

\subsection{Independent KSEs}

The substitution of the spinor (2.22) into the KSEs produces a large number of additional conditions. These can be seen either as integrability conditions along the lightcone directions, as well as integrability conditions along the mixed lightcone and $\mathcal{S}$ directions, or as KSEs along $\mathcal{S}$. A detailed analysis, presented in appendix B, of the formulae obtained reveals that the independent KSEs are those that are obtained from the naive restriction of the IIA KSEs to $\mathcal{S}$. In particular, the independent KSEs are

$$
\nabla_{i}^{( \pm)} \eta_{ \pm}=0, \quad \mathcal{A}^{( \pm)} \eta_{ \pm}=0
$$

where

$$
\nabla_{i}^{( \pm)}=\tilde{\nabla}_{i}+\Psi_{i}^{( \pm)}
$$

with

$$
\begin{aligned}
\Psi_{i}^{( \pm)}= & \left(\mp \frac{1}{4} h_{i} \mp \frac{1}{16} e^{\Phi} X_{l_{1} l_{2}} \Gamma^{l_{1} l_{2}} \Gamma_{i}+\frac{1}{8.4 !} e^{\Phi} \tilde{G}_{l_{1} l_{2} l_{3} l_{4}} \Gamma^{l_{1} l_{2} l_{3} l_{4}} \Gamma_{i}\right) \\
& +\Gamma_{11}\left(\mp \frac{1}{4} L_{i}+\frac{1}{8} \tilde{H}_{i l_{1} l_{2}} \Gamma^{l_{1} l_{2}} \pm \frac{1}{8} e^{\Phi} S \Gamma_{i}-\frac{1}{16} e^{\Phi} \tilde{F}_{l_{1} l_{2}} \Gamma^{l_{1} l_{2}} \Gamma_{i}\right)
\end{aligned}
$$

and

$$
\begin{aligned}
\mathcal{A}^{( \pm)}= & \partial_{i} \Phi \Gamma^{i}+\left(\mp \frac{1}{8} e^{\Phi} X_{l_{1} l_{2}} \Gamma^{l_{1} l_{2}}+\frac{1}{4.4 !} e^{\Phi} \tilde{G}_{l_{1} l_{2} l_{3} l_{4}} \Gamma^{l_{1} l_{2} l_{3} l_{4}}\right) \\
& +\Gamma_{11}\left( \pm \frac{1}{2} L_{i} \Gamma^{i}-\frac{1}{12} \tilde{H}_{i j k} \Gamma^{i j k} \mp \frac{3}{4} e^{\Phi} S+\frac{3}{8} e^{\Phi} \tilde{F}_{i j} \Gamma^{i j}\right) .
\end{aligned}
$$

Evidently, $\nabla^{( \pm)}$arise from the supercovariant connection while $\mathcal{A}^{( \pm)}$arise from the dilatino KSE of IIA supergravity as restricted to $\mathcal{S}$.

Furthermore, the analysis in appendix B reveals that if $\eta_{-}$solves (2.26) then

$$
\eta_{+}=\Gamma_{+} \Theta_{-} \eta_{-},
$$

also solves (2.26). This is the first indication that IIA horizons admit an even number of supersymmetries. As we shall prove, the existence of the $\eta_{+}$solution is also responsible for the $\mathfrak{s l}(2, \mathbb{R})$ symmetry of IIA horizons. 


\section{Supersymmetry enhancement}

To prove that IIA horizons always admit an even number of supersymmetries, it suffices to prove that there are as many $\eta_{+}$Killing spinors as there are $\eta_{-}$Killing spinors, i.e. that the $\eta_{+}$and $\eta_{-}$Killing spinors come in pairs. For this, we shall identify the Killing spinors with the zero modes of Dirac-like operators which depend on the fluxes and then use the index theorem to count their modes.

\subsection{Horizon Dirac equations}

We define horizon Dirac operators associated with the supercovariant derivatives following from the gravitino KSE as

$$
\mathcal{D}^{( \pm)} \equiv \Gamma^{i} \nabla_{i}^{( \pm)}=\Gamma^{i} \tilde{\nabla}_{i}+\Psi^{( \pm)},
$$

where

$$
\begin{aligned}
\Psi^{( \pm)} \equiv \Gamma^{i} \Psi_{i}^{( \pm)}= & \mp \frac{1}{4} h_{i} \Gamma^{i} \mp \frac{1}{4} e^{\Phi} X_{i j} \Gamma^{i j} \\
& +\Gamma_{11}\left( \pm \frac{1}{4} L_{i} \Gamma^{i}-\frac{1}{8} \tilde{H}_{i j k} \Gamma^{i j k} \mp e^{\Phi} S+\frac{1}{4} e^{\Phi} \tilde{F}_{i j} \Gamma^{i j}\right) .
\end{aligned}
$$

However, it turns out that it is not possible to straightforwardly formulate Lichnerowicz theorems to identify zero modes of these horizon Dirac operators with Killing spinors.

To proceed, we shall modify both the KSEs and the horizon Dirac operators. For this first observe that an equivalent set of KSEs can be chosen by redefining the supercovariant derivatives from the gravitino KSE as

$$
\hat{\nabla}_{i}^{( \pm)}=\nabla_{i}^{( \pm)}+\kappa \Gamma_{i} \mathcal{A}^{( \pm)}
$$

for some $\kappa \in \mathbb{R}$, because

$$
\hat{\nabla}_{i}^{( \pm)} \eta_{ \pm}=0, \quad \mathcal{A}^{( \pm)} \eta_{ \pm}=0 \Longleftrightarrow \nabla_{i}^{( \pm)} \eta_{ \pm}=0, \quad \mathcal{A}^{( \pm)} \eta_{ \pm}=0 .
$$

Similarly, one can modify the horizon Dirac operators as

$$
\mathscr{D}^{( \pm)}=\mathcal{D}^{( \pm)}+q \mathcal{A}^{( \pm)},
$$

for some $q \in \mathbb{R}$. Clearly, if $q=8 \kappa$, then $\mathscr{D}^{( \pm)}=\Gamma^{i} \hat{\nabla}_{i}^{( \pm)}$. However, we shall not assume this in general. As we shall see, there is an appropriate choice of $q$ and appropriate choices of $\kappa$ such that the Killing spinors can be identified with the zero modes of $\mathscr{D}^{( \pm)}$.

\subsection{A Lichnerowicz type theorem for $\mathcal{D}^{(+)}$}

First let us establish that the $\eta_{+}$Killing spinors can be identified with the zero modes of a $\mathscr{D}^{(+)}$. It is straightforward to see that if $\eta_{+}$is a Killing spinor, then $\eta_{+}$is a zero mode of $\mathscr{D}^{(+)}$. So it remains to demonstrate the converse. For this assume that $\eta_{+}$is a zero mode 
of $\mathscr{D}^{(+)}$, i.e. $\mathscr{D}^{(+)} \eta_{+}=0$. Then after some lengthy computation which utilizes the field equations and Bianchi identities, described in appendix $\mathrm{C}$, one can establish the equality

$$
\tilde{\nabla}^{i} \tilde{\nabla}_{i}\left\|\eta_{+}\right\|^{2}-\left(2 \tilde{\nabla}^{i} \Phi+h^{i}\right) \tilde{\nabla}_{i}\left\|\eta_{+}\right\|^{2}=2\left\|\hat{\nabla}^{(+)} \eta_{+}\right\|^{2}+\left(-4 \kappa-16 \kappa^{2}\right)\left\|\mathcal{A}^{(+)} \eta_{+}\right\|^{2}
$$

provided that $q=-1$. It is clear that if the last term on the right-hand-side of the above identity is positive semi-definite, then one can apply the maximum principle on $\left\|\eta_{+}\right\|^{2}$ as the fields are assumed to be smooth, and $\mathcal{S}$ compact. In particular, if

$$
-\frac{1}{4}<\kappa<0
$$

then the maximum principle implies that $\eta_{+}$are Killing spinors and $\left\|\eta_{+}\right\|=$const. Observe that if one takes $\mathscr{D}^{(+)}$with $q=-1$, then $\mathscr{D}^{(+)}=\Gamma^{i} \hat{\nabla}_{i}^{(+)}$provided that $\kappa=-1 / 8$ which lies in the range (3.7).

To summarize we have established that for $q=-1$ and $-\frac{1}{4}<\kappa<0$,

$$
\nabla_{i}^{(+)} \eta_{+}=0, \quad \mathcal{A}^{(+)} \eta_{+}=0 \Longleftrightarrow \mathscr{D}^{(+)} \eta_{+}=0
$$

Moreover $\left\|\eta_{+}\right\|^{2}$ is constant on $\mathcal{S}$.

\subsection{A Lichnerowicz type theorem for $\mathcal{D}^{(-)}$}

Next we shall establish that the $\eta_{-}$Killing spinors can also be identified with the zero modes of a modified horizon Dirac operator $\mathscr{D}^{(-)}$. It is clear that all Killing spinors $\eta_{-}$ are zero modes of $\mathscr{D}^{(-)}$. To prove the converse, suppose that $\eta_{-}$satisfies $\mathscr{D}^{(-)} \eta_{-}=0$. The proof proceeds by calculating the Laplacian of $\left\|\eta_{-}\right\|^{2}$ as described in appendix C, which requires the use of the field equations and Bianchi identies. One can then establish the formula

$$
\tilde{\nabla}^{i}\left(e^{-2 \Phi} V_{i}\right)=-2 e^{-2 \Phi}\left\|\hat{\nabla}^{(-)} \eta_{-}\right\|^{2}+e^{-2 \Phi}\left(4 \kappa+16 \kappa^{2}\right)\left\|\mathcal{A}^{(-)} \eta_{-}\right\|^{2},
$$

provided that $q=-1$, where

$$
V=-d\left\|\eta_{-}\right\|^{2}-\left\|\eta_{-}\right\|^{2} h
$$

The last term on the r.h.s. of (3.9) is negative semi-definite if $-\frac{1}{4}<\kappa<0$. Provided that this holds, on integrating (3.9) over $\mathcal{S}$ and assuming that $\mathcal{S}$ is compact and without boundary, one finds that $\hat{\nabla}^{(-)} \eta_{-}=0$ and $\mathcal{A}^{(-)} \eta_{-}=0$.

Therefore, we have shown that for $q=-1$ and $-\frac{1}{4}<\kappa<0$,

$$
\nabla_{i}^{(-)} \eta_{-}=0, \quad \mathcal{A}^{(-)} \eta_{-}=0 \Longleftrightarrow \mathscr{D}^{(-)} \eta_{-}=0
$$

This concludes the relationship between Killing spinors and zero modes of modified horizon Dirac operators. 


\subsection{Supersymmetry enhancement}

The analysis developed so far suffices to prove that IIA horizons preserve an even number of supersymmetries. Indeed, if $N_{ \pm}$is the number of $\eta_{ \pm}$Killing spinors, then the number of supersymmetries of IIA horizon is $N=N_{+}+N_{-}$. Utilizing the relation between the Killing spinors $\eta_{ \pm}$and the zero modes of the modified horizon Dirac operators $\mathscr{D}^{( \pm)}$established in the previous two sections, we have that

$$
N_{ \pm}=\operatorname{dim} \operatorname{Ker} \mathscr{D}^{( \pm)}
$$

Next let us focus on the index of the $\mathscr{D}^{(+)}$operator. As we have mentioned, the spin bundle of the spacetime $S$ decomposes on $\mathcal{S}$ as $S=S_{+} \oplus S_{-}$. Moreover, $S_{+}$and $S_{-}$are isomorphic as $\operatorname{Spin}(8)$ bundles and are associated with the Majorana non-Weyl 16 representation. Furthermore $\mathscr{D}^{(+)}: \Gamma\left(S_{+}\right) \rightarrow \Gamma\left(S_{+}\right)$, where $\Gamma\left(S_{+}\right)$are the sections of $S_{+}$ and this action does not preserve the $\operatorname{Spin}(8)$ chirality. Since the principal symbol of $\mathscr{D}^{(+)}$ is the same as the principal symbol of the standard Dirac operator acting on Majorana but not-Weyl spinors, the index vanishes ${ }^{2}[17]$. As a result, we conclude that

$$
\operatorname{dim} \operatorname{Ker} \mathscr{D}^{(+)}=\operatorname{dim} \operatorname{Ker}\left(\mathscr{D}^{(+)}\right)^{\dagger},
$$

where $\left(\mathscr{D}^{(+)}\right)^{\dagger}$ is the adjoint of $\mathscr{D}^{(+)}$. Furthermore observe that

$$
\left(e^{2 \Phi} \Gamma_{-}\right)\left(\mathscr{D}^{(+)}\right)^{\dagger}=\mathscr{D}^{(-)}\left(e^{2 \Phi} \Gamma_{-}\right), \quad(\text { for } q=-1),
$$

and so

$$
N_{-}=\operatorname{dim} \operatorname{Ker}\left(\mathscr{D}^{(-)}\right)=\operatorname{dim} \operatorname{Ker}\left(\mathscr{D}^{(+)}\right)^{\dagger} .
$$

Therefore, we conclude that $N_{+}=N_{-}$and so the number of supersymmetries of IIA horizons $N=N_{+}+N_{-}=2 N_{-}$is even. This proves the first part of the conjecture (1.1) for IIA horizons.

\section{Construction of $\eta_{+}$from $\eta_{-}$Killing spinors}

In the investigation of the integrability conditions of the KSEs, we have demonstrated that if $\eta_{-}$is a Killing spinor, then $\eta_{+}=\Gamma_{+} \Theta_{-} \eta_{-}$is also a Killing spinor, see (2.30). Since we know that the $\eta_{+}$and $\eta_{-}$Killing spinors appear in pairs, the formula (2.30) provides a way to construct the $\eta_{+}$Killing spinors from the $\eta_{-}$ones. However, this is the case provided that $\eta_{+}=\Gamma_{+} \Theta_{-} \eta_{-} \neq 0$. Here, we shall prove that for horizons with non-trivial fluxes

$$
\operatorname{Ker} \Theta_{-}=\{0\} \text {, }
$$

and so the operator $\Gamma_{+} \Theta_{-}$pairs the $\eta_{-}$with the $\eta_{+}$Killing spinors.

\footnotetext{
${ }^{2}$ This should be contrasted to IIB horizons where the horizon Dirac operators act on the Weyl spinors and map them to anti-Weyl ones. As a result, the horizon Dirac operators have the same principal symbol as the standard Dirac operator acting on the Weyl spinors and so there is a non-trivial contribution from the index.
} 
We shall prove $\operatorname{Ker} \Theta_{-}=\{0\}$ using contradiction. For this assume that $\Theta_{-}$has a non-trivial kernel, i.e. there is $\eta_{-} \neq 0$ such that

$$
\Theta_{-} \eta_{-}=0 \text {. }
$$

If this is the case, then the last integrability condition in (B.1) gives that

$$
\left\langle\eta_{-},\left(-\frac{1}{2} \Delta-\frac{1}{8} d h_{i j} \Gamma^{i j}+\frac{1}{8} M_{i j} \Gamma^{i j} \Gamma_{11}-\frac{1}{4} e^{\Phi} T_{i} \Gamma^{i} \Gamma_{11}-\frac{1}{24} e^{\Phi} Y_{i j k} \Gamma^{i j k}\right) \eta_{-}\right\rangle=0 .
$$

This in turn implies that

$$
\Delta\left\langle\eta_{-}, \eta_{-}\right\rangle=0
$$

and hence

$$
\Delta=0,
$$

as $\eta_{-}$is no-where vanishing.

Next the gravitino KSE $\nabla^{(-)} \eta_{-}=0$ implies that

$$
\begin{aligned}
\tilde{\nabla}_{i}\left\langle\eta_{-}, \eta_{-}\right\rangle= & -\frac{1}{2} h_{i}\left\langle\eta_{-}, \eta_{-}\right\rangle+\left\langle\eta_{-},\left(\frac{1}{4} e^{\Phi} X_{i \ell} \Gamma^{\ell}-\frac{1}{96} e^{\Phi} \tilde{G}_{\ell_{1} \ell_{2} \ell_{3} \ell_{4}} \Gamma_{i}^{\ell_{1} \ell_{2} \ell_{3} \ell_{4}}\right) \eta_{-}\right\rangle \\
& +\left\langle\eta_{-}, \Gamma_{11}\left(-\frac{1}{2} L_{i}+\frac{1}{8} e^{\Phi} \tilde{F}_{\ell_{1} \ell_{2}} \Gamma_{i}^{\ell_{1} \ell_{2}}\right) \eta_{-}\right\rangle
\end{aligned}
$$

which can be simplified further using

$$
\begin{aligned}
\left\langle\eta_{-}, \Gamma_{i} \Theta_{-} \eta_{-}\right\rangle= & \frac{1}{4} h_{i}\left\langle\eta_{-}, \eta_{-}\right\rangle+\left\langle\eta_{-},\left(\frac{1}{8} e^{\Phi} X_{i \ell} \Gamma^{\ell}-\frac{1}{192} e^{\Phi} \tilde{G}_{\ell_{1} \ell_{2} \ell_{3} \ell_{4}} \Gamma_{i}^{\ell_{1} \ell_{2} \ell_{3} \ell_{4}}\right) \eta_{-}\right\rangle \\
& +\left\langle\eta_{-}, \Gamma_{11}\left(-\frac{1}{4} L_{i}+\frac{1}{16} e^{\Phi} \tilde{F}_{\ell_{1} \ell_{2}} \Gamma_{i}^{\ell_{1} \ell_{2}}\right) \eta_{-}\right\rangle=0,
\end{aligned}
$$

to yield

$$
\tilde{\nabla}_{i}\left\|\eta_{-}\right\|^{2}=-h_{i}\left\|\eta_{-}\right\|^{2}
$$

As $\eta_{-}$is no-where zero, this implies that

$$
d h=0 .
$$

Substituting, $\Delta=0$ and $d h=0$ into (A.5), we find that

$$
M=d_{h} L=0, \quad T=d_{h} S=0, \quad Y=d_{h} X-L \wedge \tilde{F}-S \tilde{H}=0,
$$

as well. Returning to (4.8), on taking the divergence, and using (2.18) to eliminate the $\tilde{\nabla}^{i} h_{i}$ term, one obtains

$\tilde{\nabla}^{i} \tilde{\nabla}_{i}\left\|\eta_{-}\right\|^{2}=2 \tilde{\nabla}^{i} \Phi \tilde{\nabla}_{i}\left\|\eta_{-}\right\|^{2}+\left(L^{2}+\frac{1}{2} e^{2 \Phi} S^{2}+\frac{1}{4} e^{2 \Phi} X^{2}+\frac{1}{4} e^{2 \Phi} \tilde{F}^{2}+\frac{1}{48} e^{2 \Phi} \tilde{G}^{2}\right)\left\|\eta_{-}\right\|^{2}$.

Applying the maximum principle on $\left\|\eta_{-}\right\|^{2}$ we conclude that all the fluxes apart from the dilaton $\Phi$ and $\tilde{H}$ vanish and $\left\|\eta_{-}\right\|$is constant. The latter together with (4.8) imply that $h=0$. 
Next applying the maximum principle to the dilaton field equation (2.17), we conclude that the dilaton is constant and $\tilde{H}=0$. Combining all the results so far, we conclude that all the fluxes vanish which is a contradiction to the assumption that not all of the fluxes vanish. This establishes (4.1).

Furthermore, the horizons for which $\Theta_{-} \eta_{-}=0\left(\eta_{-} \neq 0\right)$ are all local products $\mathbb{R}^{1,1} \times \mathcal{S}$, where $\mathcal{S}$ up to a discrete identification is a product of Ricci flat Berger manifolds. Thus $\mathcal{S}$ has holonomy, $\operatorname{Spin}(7)$ or $\mathrm{SU}(4)$ or $\mathrm{Sp}(2)$ as an irreducible manifold, and $G_{2}$ or $\mathrm{SU}(3)$ or $\operatorname{Sp}(1) \times \operatorname{Sp}(1)$ or $\operatorname{Sp}(1)$ or $\{1\}$ as a reducible one.

\section{$5 \quad$ The $\mathfrak{s l}(2, \mathbb{R})$ symmetry of IIA horizons}

It remains to prove the second part of the conjecture that all IIA horizons with nontrivial fluxes admit an $\mathfrak{s l}(2, \mathbb{R})$ symmetry subalgebra. As we shall demonstrate, this in fact is a consequence of our previous result that all IIA horizons admit an even number of supersymmetries. The proof is very similar to that already given in the context of M-horizons in [3], so we shall be brief.

\section{$5.1 \quad$ Killing vectors}

To begin, first note that the Killing spinor $\epsilon$ on the spacetime can be expressed in terms of $\eta_{ \pm}$as

$$
\epsilon=\eta_{+}+u \Gamma_{+} \Theta_{-} \eta_{-}+\eta_{-}+r \Gamma_{-} \Theta_{+} \eta_{+}+r u \Gamma_{-} \Theta_{+} \Gamma_{+} \Theta_{-} \eta_{-},
$$

which is derived after collecting the results of section 2.3 .

Since the $\eta_{-}$and $\eta_{+}$Killing spinors appear in pairs for supersymmetric IIA horizons, let us choose a $\eta_{-}$Killing spinor. Then from the results of the previous section, horizons with non-trivial fluxes also admit $\eta_{+}=\Gamma_{+} \Theta_{-} \eta_{-}$as a Killing spinors. Using $\eta_{-}$and $\eta_{+}=$ $\Gamma_{+} \Theta_{-} \eta_{-}$, one can construct two linearly independent Killing spinors on the spacetime as

$$
\epsilon_{1}=\eta_{-}+u \eta_{+}+r u \Gamma_{-} \Theta_{+} \eta_{+}, \quad \epsilon_{2}=\eta_{+}+r \Gamma_{-} \Theta_{+} \eta_{+} .
$$

To continue, it is known from the general theory of supersymmetric IIA backgrounds that for any Killing spinors $\zeta_{1}$ and $\zeta_{2}$ the dual vector field of the 1-form bilinear

$$
K\left(\zeta_{1}, \zeta_{2}\right)=\left\langle\left(\Gamma_{+}-\Gamma_{-}\right) \zeta_{1}, \Gamma_{a} \zeta_{2}\right\rangle e^{a},
$$

is a Killing vector and leaves invariant all the other fields of the theory. Evaluating, the 1-form bilinears of the Killing spinor $\epsilon_{1}$ and $\epsilon_{2}$, we find that

$$
\begin{aligned}
K_{1}\left(\epsilon_{1}, \epsilon_{2}\right)= & \left(2 r\left\langle\Gamma_{+} \eta_{-}, \Theta_{+} \eta_{+}\right\rangle+u^{2} r \Delta\left\|\eta_{+}\right\|^{2}\right) \mathbf{e}^{+}-2 u\left\|\eta_{+}\right\|^{2} \mathbf{e}^{-}+V_{i} \mathbf{e}^{i}, \\
K_{2}\left(\epsilon_{2}, \epsilon_{2}\right)= & r^{2} \Delta\left\|\eta_{+}\right\|^{2} \mathbf{e}^{+}-2\left\|\eta_{+}\right\|^{2} \mathbf{e}^{-} \\
K_{3}\left(\epsilon_{1}, \epsilon_{1}\right)= & \left(2\left\|\eta_{-}\right\|^{2}+4 r u\left\langle\Gamma_{+} \eta_{-}, \Theta_{+} \eta_{+}\right\rangle+r^{2} u^{2} \Delta\left\|\eta_{+}\right\|^{2}\right) \mathbf{e}^{+} \\
& -2 u^{2}\left\|\eta_{+}\right\|^{2} \mathbf{e}^{-}+2 u V_{i} \mathbf{e}^{i}
\end{aligned}
$$

where we have set

$$
V_{i}=\left\langle\Gamma_{+} \eta_{-}, \Gamma_{i} \eta_{+}\right\rangle
$$


Moreover, we have used the identities

$$
-\Delta\left\|\eta_{+}\right\|^{2}+4\left\|\Theta_{+} \eta_{+}\right\|^{2}=0, \quad\left\langle\eta_{+}, \Gamma_{i} \Theta_{+} \eta_{+}\right\rangle=0,
$$

which follow from the first integrability condition in (B.1), $\left\|\eta_{+}\right\|=$const and the KSEs of $\eta_{+}$.

\subsection{The geometry of $\mathcal{S}$}

First suppose that $V \neq 0$. Then the conditions $\mathcal{L}_{K_{a}} g=0$ and $\mathcal{L}_{K_{a}} F=0, a=1,2,3$, where $F$ denotes collectively all the fluxes of IIA supergravity, imply that

$$
\begin{aligned}
\tilde{\nabla}_{(i} V_{j)} & =0, \quad \tilde{\mathcal{L}}_{V} h=\tilde{\mathcal{L}}_{V} \Delta=0, \quad \tilde{\mathcal{L}}_{V} \Phi=0, \\
\tilde{\mathcal{L}}_{V} X & =\tilde{\mathcal{L}}_{V} \tilde{G}=\tilde{\mathcal{L}}_{V} L=\tilde{\mathcal{L}}_{V} \tilde{H}=\tilde{\mathcal{L}}_{V} S=\tilde{\mathcal{L}}_{V} \tilde{F}=0,
\end{aligned}
$$

i.e. $V$ is an isometry of $\mathcal{S}$ and leaves all the fluxes on $\mathcal{S}$ invariant. In addition, one also finds the useful identities

$$
\begin{aligned}
& -2\left\|\eta_{+}\right\|^{2}-h_{i} V^{i}+2\left\langle\Gamma_{+} \eta_{-}, \Theta_{+} \eta_{+}\right\rangle=0, \quad i_{V}(d h)+2 d\left\langle\Gamma_{+} \eta_{-}, \Theta_{+} \eta_{+}\right\rangle=0, \\
& 2\left\langle\Gamma_{+} \eta_{-}, \Theta_{+} \eta_{+}\right\rangle-\Delta\left\|\eta_{-}\right\|^{2}=0, \quad V+\left\|\eta_{-}\right\|^{2} h+d\left\|\eta_{-}\right\|^{2}=0,
\end{aligned}
$$

which imply that $\mathcal{L}_{V}\left\|\eta_{-}\right\|^{2}=0$. There are further restrictions on the geometry of $\mathcal{S}$ which will be explored elsewhere.

A special case arises for $V=0$ where the group action generated by $K_{1}, K_{2}$ and $K_{3}$ has only 2-dimensional orbits. A direct substitution of this condition in (5.8) reveals that

$$
\Delta\left\|\eta_{-}\right\|^{2}=2\left\|\eta_{+}\right\|^{2}, \quad h=\Delta^{-1} d \Delta .
$$

Since $d h=0$ and $h$ is exact such horizons are static and a coordinate transformation $r \rightarrow \Delta r$ reveals that the horizon geometry is a warped product of $A d S_{2}$ with $\mathcal{S}, A d S_{2} \times{ }_{w} \mathcal{S}$.

\section{$\mathbf{5 . 3} \mathfrak{s l}(2, \mathbb{R})$ symmetry of IIA-horizons}

To uncover the $\mathfrak{s l}(2, \mathbb{R})$ symmetry of IIA horizons it remains to compute the Lie bracket algebra of the vector fields associated to the 1-forms $K_{1}, K_{2}$ and $K_{3}$. For this note that these vector fields can be expressed as

$$
\begin{aligned}
& K_{1}=-2 u\left\|\eta_{+}\right\|^{2} \partial_{u}+2 r\left\|\eta_{+}\right\|^{2} \partial_{r}+V^{i} \tilde{\partial}_{i}, \\
& K_{2}=-2\left\|\eta_{+}\right\|^{2} \partial_{u}, \\
& K_{3}=-2 u^{2}\left\|\eta_{+}\right\|^{2} \partial_{u}+\left(2\left\|\eta_{-}\right\|^{2}+4 r u\left\|\eta_{+}\right\|^{2}\right) \partial_{r}+2 u V^{i} \tilde{\partial}_{i},
\end{aligned}
$$

where we have used the same symbol for the 1-forms and the associated vector fields. These expressions are similar to those we have obtained for M-horizons in [3] apart form the range of the index $i$ which is different. Using the various identities we have obtained, a direct computation reveals that the Lie bracket algebra is

$$
\left[K_{1}, K_{2}\right]=2\left\|\eta_{+}\right\|^{2} K_{2}, \quad\left[K_{2}, K_{3}\right]=-4\left\|\eta_{+}\right\|^{2} K_{1}, \quad\left[K_{3}, K_{1}\right]=2\left\|\eta_{+}\right\|^{2} K_{3},
$$

which is isomorphic to $\mathfrak{s l}(2, \mathbb{R})$. This proves the second part of the conjecture and completes the analysis. 


\section{Conclusions}

We have demonstrated that smooth IIA horizons with compact spatial sections, without boundary, always admit an even number of supersymmetries. In addition, those with non-trivial fluxes admit an $\mathfrak{s l}(2, \mathbb{R})$ symmetry subalgebra.

The above result together with those obtained in [2,3] and [1] provide further evidence to support the conjecture of [1] regarding the (super)symmetries of supergravity horizons. It also emphases that the (super)symmetry enhancement that is observed near the horizons of supersymmetric black holes is a consequence of the smoothness of the fields.

Apart from exhibiting an $\mathfrak{s l}(2, \mathbb{R})$ symmetry, IIA horizons are further geometrically restricted. This is because we have not explored all the restrictions imposed by the KSEs and the field equations of the theory - in this paper we only explored enough to establish the $\mathfrak{s l}(2, \mathbb{R})$ symmetry. However, the understanding of the horizons admitting two supersymmetries is within the capability of the technology developed so far for the classification of supersymmetric IIA backgrounds [19] and it will be explored elsewhere. The understanding of all IIA horizons is a more involved problem. As such spaces preserve an even number of supersymmetries and there are no IIA horizons with non-trivial fluxes preserving 32 supersymmetries, which follows from the classification of maximally supersymmetric backgrounds in [18], there are potentially 15 different cases to examine. Of course, all IIA horizons preserving more than 16 supersymmetries are homogenous spaces as a consequence of the results of [20]. It is also very likely that there are no IIA horizons preserving 28 and 30 supersymmetries in analogy with a similar result in IIB [21-23]. However to prove this, it is required to extend the IIB classification results to IIA supergravity, see also [24].

We expect that our results on IIA horizons can be extended to massive IIA supergravity [15]. This will be reported elsewhere.

\section{Acknowledgments}

UG is supported by the Knut and Alice Wallenberg Foundation. GP is partially supported by the STFC grant ST/J002798/1. JG is supported by the STFC grant, ST/1004874/1. JG would like to thank the Department of Mathematical Sciences, University of Liverpool for hospitality during which part of this work was completed. U.K. is supported by a STFC PhD fellowship.

\section{A Horizon Bianchi identities and field equations}

We remark that there are a number of additional Bianchi identities, which are

$$
\begin{aligned}
d T+S d h+d S \wedge h & =0, \\
d M+L \wedge d h-h \wedge d L & =0, \\
d Y+d h \wedge X-h \wedge d X+h \wedge(S \tilde{H}+\tilde{F} \wedge L)+T \wedge \tilde{H}+\tilde{F} \wedge M & =0 .
\end{aligned}
$$

However, these Bianchi identities are implied by those in (2.11). 
There is also a number of additional field equations given by

$$
\begin{aligned}
&-\tilde{\nabla}^{i} T_{i}+h^{i} T_{i}-\frac{1}{2} d h^{i j} \tilde{F}_{i j}-\frac{1}{2} X_{i j} M^{i j}-\frac{1}{6} Y_{i j k} \tilde{H}^{i j k}=0, \\
&-\tilde{\nabla}^{i}\left(e^{-2 \Phi} M_{i k}\right)+e^{-2 \Phi} h^{i} M_{i k}-\frac{1}{2} e^{-2 \Phi} d h^{i j} \tilde{H}_{i j k}-T^{i} X_{i k}-\frac{1}{2} \tilde{F}^{i j} Y_{i j k} \\
&-\frac{1}{144} \epsilon_{k}{ }^{\ell_{1} \ell_{2} \ell_{3} \ell_{4} \ell_{5} \ell_{6} \ell_{7}} Y_{\ell_{1} \ell_{2} \ell_{3}} \tilde{G}_{\ell_{4} \ell_{5} \ell_{6} \ell_{7}}=0, \\
&-\tilde{\nabla}^{i} Y_{i m n}+h^{i} Y_{i m n}-\frac{1}{2} d h^{i j} \tilde{G}_{i j m n}+\frac{1}{36} \epsilon_{m n}{ }^{\ell_{1} \ell_{2} \ell_{3} \ell_{4} \ell_{5} \ell_{6}} Y_{\ell_{1} \ell_{2} \ell_{3}} \tilde{H}_{\ell_{4} \ell_{5} \ell_{6}} \\
&+\frac{1}{48} \epsilon_{m n}{ }^{\ell_{1} \ell_{2} \ell_{3} \ell_{4} \ell_{5} \ell_{6}} \tilde{G}_{\ell_{1} \ell_{2} \ell_{3} \ell_{4}} M_{\ell_{5} \ell_{6}}=0,
\end{aligned}
$$

corresponding to equations obtained from the + component of (2.6), the $k$ component of (2.7) and the $m n$ component of (2.8) respectively. However, (A.2), (A.3) and (A.4) are implied by (2.12)-(2.16) together with the Bianchi identities (2.11).

Note also that the ++ and $+i$ components of the Einstein equation, which are

$$
\begin{aligned}
\frac{1}{2} \tilde{\nabla}^{i} \tilde{\nabla}_{i} \Delta-\frac{3}{2} h^{i} \tilde{\nabla}_{i} \Delta-\frac{1}{2} \Delta \tilde{\nabla}^{i} h_{i}+\Delta h^{2}+\frac{1}{4} d h_{i j} d h^{i j}= & \left(\tilde{\nabla}^{i} \Delta-\Delta h^{i}\right) \tilde{\nabla}_{i} \Phi+\frac{1}{4} M_{i j} M^{i j} \\
& +\frac{1}{2} e^{2 \Phi} T_{i} T^{i}+\frac{1}{12} e^{2 \Phi} Y_{i j k} Y^{i j k}
\end{aligned}
$$

and

$$
\begin{aligned}
\frac{1}{2} \tilde{\nabla}^{j} d h_{i j}-d h_{i j} h^{j}-\tilde{\nabla}_{i} \Delta+\Delta h_{i}= & d h_{i}{ }^{j} \tilde{\nabla}_{j} \Phi-\frac{1}{2} M_{i}{ }^{j} L_{j}+\frac{1}{4} M_{\ell_{1} \ell_{2}} \tilde{H}_{i}{ }^{\ell_{1} \ell_{2}}-\frac{1}{2} e^{2 \Phi} S T_{i} \\
& +\frac{1}{2} e^{2 \Phi} T^{j} \tilde{F}_{i j}-\frac{1}{4} e^{2 \Phi} Y_{i}^{\ell_{1} \ell_{2}} X_{\ell_{1} \ell_{2}}+\frac{1}{12} e^{2 \Phi} Y_{\ell_{1} \ell_{2} \ell_{3}} \tilde{G}_{i}{ }^{\ell_{1} \ell_{2} \ell_{3}}
\end{aligned}
$$

are implied by (2.17), (2.18), (2.19), together with (2.12)-(2.16), and the Bianchi identities (2.11).

\section{B Integrability conditions and KSEs}

Substituting the solution of the KSEs along the lightcone directions (2.23) back into the gravitino KSE (2.20) and appropriately expanding in the $r, u$ coordinates, we find that for the $\mu= \pm$ components, one obtains the additional conditions

$$
\begin{gathered}
\left(\frac{1}{2} \Delta-\frac{1}{8}(d h)_{i j} \Gamma^{i j}+\frac{1}{8} M_{i j} \Gamma_{11} \Gamma^{i j}\right. \\
\left.+2\left(\frac{1}{4} h_{i} \Gamma^{i}-\frac{1}{4} L_{i} \Gamma_{11} \Gamma^{i}-\frac{1}{16} e^{\Phi} \Gamma_{11}\left(-2 S+\tilde{F}_{i j} \Gamma^{i j}\right)-\frac{1}{8 \cdot 4 !} e^{\Phi}\left(12 X_{i j} \Gamma^{i j}-\tilde{G}_{i j k l} \Gamma^{i j k l}\right)\right) \Theta_{+}\right) \phi_{+}=0 \\
\left(\frac{1}{4} \Delta h_{i} \Gamma^{i}-\frac{1}{4} \partial_{i} \Delta \Gamma^{i}+\left(-\frac{1}{8}(d h)_{i j} \Gamma^{i j}-\frac{1}{8} M_{i j} \Gamma^{i j} \Gamma_{11}-\frac{1}{4} e^{\Phi} T_{i} \Gamma^{i} \Gamma_{11}+\frac{1}{24} e^{\Phi} Y_{i j k} \Gamma^{i j k}\right) \Theta_{+}\right) \phi_{+}=0 \\
\left(-\frac{1}{2} \Delta-\frac{1}{8}(d h)_{i j} \Gamma^{i j}+\frac{1}{8} M_{i j} \Gamma^{i j} \Gamma_{11}-\frac{1}{4} e^{\Phi} T_{i} \Gamma^{i} \Gamma_{11}-\frac{1}{24} e^{\Phi} Y_{i j k} \Gamma^{i j k}\right. \\
\left.+2\left(-\frac{1}{4} h_{i} \Gamma^{i}-\frac{1}{4} \Gamma_{11} L_{i} \Gamma^{i}+\frac{1}{16} e^{\phi} \Gamma_{11}\left(2 S+\tilde{F}_{i j} \Gamma^{i j}\right)-\frac{1}{8 \cdot 4 !} e^{\phi}\left(12 X_{i j} \Gamma^{i j}+\tilde{G}_{i j k l} \Gamma^{i j k l}\right)\right) \Theta_{-}\right) \phi_{-}=0 .
\end{gathered}
$$


Similarly the $\mu=i$ component of the gravitino KSEs gives

$$
\begin{gathered}
\tilde{\nabla}_{i} \phi_{ \pm} \mp \frac{1}{4} h_{i} \phi_{ \pm} \mp \frac{1}{4} \Gamma_{11} L_{i} \phi_{ \pm}+\frac{1}{8} \Gamma_{11} \tilde{H}_{i j k} \Gamma^{j k} \phi_{ \pm} \\
-\frac{1}{16} e^{\Phi} \Gamma_{11}\left(\mp 2 S+\tilde{F}_{k l} \Gamma^{k l}\right) \Gamma_{i} \phi_{ \pm}+\frac{1}{8 \cdot 4 !} e^{\Phi}\left(\mp 12 X_{k l} \Gamma^{k l}+\tilde{G}_{j_{1} j_{2} j_{3} j_{4}} \Gamma^{j_{1} j_{2} j_{3} j_{4}}\right) \Gamma_{i} \phi_{ \pm}=0
\end{gathered}
$$

and

$$
\begin{gathered}
\tilde{\nabla}_{i} \tau_{+}+\left(-\frac{3}{4} h_{i}-\frac{1}{16} e^{\Phi} X_{l_{1} l_{2}} \Gamma^{l_{1} l_{2}} \Gamma_{i}-\frac{1}{8 \cdot 4 !} e^{\Phi} \tilde{G}_{l_{1} \cdots l_{4}} \Gamma^{l_{1} \cdots l_{4}} \Gamma_{i}\right. \\
\left.-\Gamma_{11}\left(\frac{1}{4} L_{i}+\frac{1}{8} \tilde{H}_{i j k} \Gamma^{j k}+\frac{1}{8} e^{\Phi} S \Gamma_{i}+\frac{1}{16} e^{\Phi} \tilde{F}_{l_{1} l_{2}} \Gamma^{l_{1} l_{2}} \Gamma_{i}\right)\right) \tau_{+} \\
+\left(-\frac{1}{4}(d h)_{i j} \Gamma^{j}-\frac{1}{4} M_{i j} \Gamma^{j} \Gamma_{11}+\frac{1}{8} e^{\Phi} T_{j} \Gamma^{j} \Gamma_{i} \Gamma_{11}+\frac{1}{48} e^{\Phi} Y_{l_{1} l_{2} l_{3}} \Gamma^{l_{1} l_{2} l_{3}} \Gamma_{i}\right) \phi_{+}=0
\end{gathered}
$$

where we have set

$$
\tau_{+}=\Theta_{+} \phi_{+} .
$$

All the additional conditions above can be viewed as integrability conditions along the lightcone and mixed lightcone and $\mathcal{S}$ directions. We shall demonstrate that upon using the field equations and the Bianchi identities, the only independent conditions are (2.26).

\section{B.1 Dilatino KSE}

Substituting the solution of the KSEs (2.23) into the dilatino KSE (2.21) and expanding appropriately in the $r, u$ coordinates, one obtains the following additional conditions

$$
\begin{gathered}
\partial_{i} \Phi \Gamma^{i} \phi_{ \pm}-\frac{1}{12} \Gamma_{11}\left(\mp 6 L_{i} \Gamma^{i}+\tilde{H}_{i j k} \Gamma^{i j k}\right) \phi_{ \pm}+\frac{3}{8} e^{\Phi} \Gamma_{11}\left(\mp 2 S+\tilde{F}_{i j} \Gamma^{i j}\right) \phi_{ \pm} \\
+\frac{1}{4 \cdot 4 !} e^{\Phi}\left(\mp 12 X_{i j} \Gamma^{i j}+\tilde{G}_{j_{1} j_{2} j_{3} j_{4}} \Gamma^{j_{1} j_{2} j_{3} j_{4}}\right) \phi_{ \pm}=0 \\
-\left(\partial_{i} \Phi \Gamma^{i}+\frac{1}{12} \Gamma_{11}\left(6 L_{i} \Gamma^{i}+\tilde{H}_{i j k} \Gamma^{i j k}\right)+\frac{3}{8} e^{\Phi} \Gamma_{11}\left(2 S+\tilde{F}_{i j} \Gamma^{i j}\right)\right. \\
\left.-\frac{1}{4 \cdot 4 !} e^{\Phi}\left(12 X_{i j} \Gamma^{i j}+\tilde{G}_{i j k l} \Gamma^{i j k l}\right)\right) \tau_{+} \\
+\left(\frac{1}{4} M_{i j} \Gamma^{i j} \Gamma_{11}+\frac{3}{4} e^{\Phi} T_{i} \Gamma^{i} \Gamma_{11}+\frac{1}{24} e^{\Phi} Y_{i j k} \Gamma^{i j k}\right) \phi_{+}=0 .
\end{gathered}
$$

We shall show that the only independent ones are those in (2.26).

\section{B.2 Independent KSEs}

It is well known that the KSEs imply some of the Bianchi identities and field equations of a theory. Because of this, to find solutions it is customary to solve the KSEs and then impose the remaining field equations and Bianchi identities. However, we shall not do this here because of the complexity of solving the KSEs (B.1), (B.2), (B.5), and (B.8) which contain 
the $\tau_{+}$spinor as expressed in (B.6). Instead, we shall first show that all the KSEs which contain $\tau_{+}$are actually implied from those containing $\phi_{+}$, i.e. (B.4) and (B.7), and some of the field equations and Bianchi identities. Then we also show that (B.3) and the terms linear in $\mathrm{u}$ from the + components of (B.4) and (B.7) are implied by the field equations, Bianchi identities and the - components of (B.4) and (B.7).

\section{B.2.1 The (B.5) condition}

The (B.5) component of the KSEs is implied by (B.4), (B.6) and (B.7) together with a number of field equations and Bianchi identities. First evaluate the 1.h.s. of (B.5) by substituting in (B.6) to eliminate $\tau_{+}$, and use (B.4) to evaluate the supercovariant derivative of $\phi_{+}$. Also, using (B.4) one can compute

$$
\begin{aligned}
& \left(\tilde{\nabla}_{j} \tilde{\nabla}_{i}-\tilde{\nabla}_{i} \tilde{\nabla}_{j}\right) \phi_{+}=\frac{1}{4} \tilde{\nabla}_{j}\left(h_{i}\right) \phi_{+}+\frac{1}{4} \Gamma_{11} \tilde{\nabla}_{j}\left(L_{i}\right) \phi_{+}-\frac{1}{8} \Gamma_{11} \tilde{\nabla}_{j}\left(\tilde{H}_{i l_{1} l_{2}}\right) \Gamma^{l_{1} l_{2}} \phi_{+} \\
& +\frac{1}{16} e^{\Phi} \Gamma_{11}\left(-2 \tilde{\nabla}_{j}(S)+\tilde{\nabla}_{j}\left(\tilde{F}_{k l}\right) \Gamma^{k l}\right) \Gamma_{i} \phi_{+} \\
& -\frac{1}{8 \cdot 4 !} e^{\Phi}\left(-12 \tilde{\nabla}_{j}\left(X_{k l}\right) \Gamma^{k l}+\tilde{\nabla}_{j}\left(\tilde{G}_{j_{1} j_{2} j_{3} j_{4}}\right) \Gamma^{j_{1} j_{2} j_{3} j_{4}}\right) \Gamma_{i} \phi_{+} \\
& +\frac{1}{16} \tilde{\nabla}_{j} \Phi e^{\Phi} \Gamma_{11}\left(-2 S+\tilde{F}_{k l} \Gamma^{k l}\right) \Gamma_{i} \phi_{+} \\
& -\frac{1}{8 \cdot 4 !} \tilde{\nabla}_{j} \Phi e^{\Phi}\left(-12 X_{k l} \Gamma^{k l}+\tilde{G}_{j_{1} j_{2} j_{3} j_{4}} \Gamma^{j_{1} j_{2} j_{3} j_{4}}\right) \Gamma_{i} \phi_{+} \\
& +\left(\frac{1}{4} h_{i}+\frac{1}{4} \Gamma_{11} L_{i}-\frac{1}{8} \Gamma_{11} \tilde{H}_{i j k} \Gamma^{j k}+\frac{1}{16} e^{\Phi} \Gamma_{11}\left(-2 S+\tilde{F}_{k l} \Gamma^{k l}\right) \Gamma_{i}\right. \\
& \left.-\frac{1}{8 \cdot 4 !} e^{\Phi}\left(-12 X_{k l} \Gamma^{k l}+\tilde{G}_{j_{1} j_{2} j_{3} j_{4}} \Gamma^{j_{1} j_{2} j_{3} j_{4}}\right) \Gamma_{i}\right) \tilde{\nabla}_{j} \phi_{+}-(i \leftrightarrow j) .
\end{aligned}
$$

Then consider the following, where the first terms cancels from the definition of curvature,

$$
\left(\frac{1}{4} \tilde{R}_{i j} \Gamma^{j}-\frac{1}{2} \Gamma^{j}\left(\tilde{\nabla}_{j} \tilde{\nabla}_{i}-\tilde{\nabla}_{i} \tilde{\nabla}_{j}\right)\right) \phi_{+}+\frac{1}{2} \tilde{\nabla}_{i}\left(\mathcal{A}_{1}\right)+\frac{1}{2} \Psi_{i} \mathcal{A}_{1}=0
$$

where

$$
\begin{aligned}
\mathcal{A}_{1}= & \partial_{i} \Phi \Gamma^{i} \phi_{+}-\frac{1}{12} \Gamma_{11}\left(-6 L_{i} \Gamma^{i}+\tilde{H}_{i j k} \Gamma^{i j k}\right) \phi_{+}+\frac{3}{8} e^{\Phi} \Gamma_{11}\left(-2 S+\tilde{F}_{i j} \Gamma^{i j}\right) \phi_{+} \\
& +\frac{1}{4 \cdot 4 !} e^{\Phi}\left(-12 X_{i j} \Gamma^{i j}+\tilde{G}_{j_{1} j_{2} j_{3} j_{4}} \Gamma^{j_{1} j_{2} j_{3} j_{4}}\right) \phi_{+}
\end{aligned}
$$

and

$$
\Psi_{i}=-\frac{1}{4} h_{i}+\Gamma_{11}\left(\frac{1}{4} L_{i}-\frac{1}{8} \tilde{H}_{i j k} \Gamma^{j k}\right) .
$$

The expression in (B.11) vanishes on making use of (B.7), as $\mathcal{A}_{1}=0$ is equivalent to the + component of (B.7). However a non-trivial identity is obtained by using (B.9) in (B.10), and expanding out the $\mathcal{A}_{1}$ terms. Then, on adding (B.10) to the l.h.s. of (B.5), with $\tau_{+}$ 
eliminated in favour of $\eta_{+}$as described above, one obtains the following

$$
\begin{gathered}
\frac{1}{4}\left(\tilde{R}_{i j}+\tilde{\nabla}_{i i} h_{j}-\frac{1}{2} h_{i} h_{j}+2 \tilde{\nabla}_{i} \tilde{\nabla}_{j} \Phi+\frac{1}{2} L_{i} L_{j}-\frac{1}{4} \tilde{H}_{i l_{1} l_{2}} \tilde{H}_{j} l_{1} l_{2}\right. \\
-\frac{1}{2} e^{2 \Phi} \tilde{F}_{i l} \tilde{F}_{j}^{l}+\frac{1}{8} e^{2 \Phi} \tilde{F}_{l_{1} l_{2}} \tilde{F}^{l_{1} l_{2}} \delta_{i j}+\frac{1}{2} e^{2 \Phi} X_{i l} X_{j}{ }^{l}-\frac{1}{8} e^{2 \Phi} X_{l_{1} l_{2}} X^{l_{1} l_{2}} \delta_{i j} \\
\left.-\frac{1}{12} e^{2 \Phi} \tilde{G}_{i \ell_{1} \ell_{2} \ell_{3}} \tilde{G}_{j}^{\ell_{1} \ell_{2} \ell_{3}}+\frac{1}{96} e^{2 \Phi} \tilde{G}_{\ell_{1} \ell_{2} \ell_{3} \ell_{4}} \tilde{G}^{\ell_{1} \ell_{2} \ell_{3} \ell_{4}} \delta_{i j}-\frac{1}{4} e^{2 \Phi} S^{2} \delta_{i j}\right) \Gamma^{j}=0 .
\end{gathered}
$$

This vanishes identically on making use of the Einstein equation (2.19). Therefore it follows that (B.5) is implied by the + component of (B.4), (B.6) and (B.7), the Bianchi identities (2.11) and the gauge field equations (2.12)-(2.16).

\section{B.2.2 The (B.8) condition}

Let us define

$$
\begin{aligned}
\mathcal{A}_{2}= & -\left(\partial_{i} \Phi \Gamma^{i}+\frac{1}{12} \Gamma_{11}\left(6 L_{i} \Gamma^{i}+\tilde{H}_{i j k} \Gamma^{i j k}\right)+\frac{3}{8} e^{\Phi} \Gamma_{11}\left(2 S+\tilde{F}_{i j} \Gamma^{i j}\right)\right. \\
& \left.-\frac{1}{4 \cdot 4 !} e^{\Phi}\left(12 X_{i j} \Gamma^{i j}+\tilde{G}_{i j k l} \Gamma^{i j k l}\right)\right) \tau_{+} \\
& +\left(\frac{1}{4} M_{i j} \Gamma^{i j} \Gamma_{11}+\frac{3}{4} e^{\Phi} T_{i} \Gamma^{i} \Gamma_{11}+\frac{1}{24} e^{\Phi} Y_{i j k} \Gamma^{i j k}\right) \phi_{+},
\end{aligned}
$$

where $\mathcal{A}_{2}$ equals the expression in (B.8). One obtains the following identity

$$
\mathcal{A}_{2}=-\frac{1}{2} \Gamma^{i} \tilde{\nabla}_{i} \mathcal{A}_{1}+\Psi_{1} \mathcal{A}_{1}
$$

where

$$
\begin{aligned}
\Psi_{1}= & \tilde{\nabla}_{i} \Phi \Gamma^{i}+\frac{3}{8} h_{i} \Gamma^{i}+\frac{1}{16} e^{\Phi} X_{l_{1} l_{2}} \Gamma^{l_{1} l_{2}}-\frac{1}{192} e^{\Phi} \tilde{G}_{l_{1} l_{2} l_{3} l_{4}} \Gamma^{l_{1} l_{2} l_{3} l_{4}} \\
& +\Gamma_{11}\left(\frac{1}{48} \tilde{H}_{l_{1} l_{2} l_{3}} \Gamma^{l_{1} l_{2} l_{3}}-\frac{1}{8} L_{i} \Gamma^{i}+\frac{1}{16} e^{\Phi} \tilde{F}_{l_{1} l_{2}} \Gamma^{l_{1} l_{2}}-\frac{1}{8} e^{\Phi} S\right)
\end{aligned}
$$

We have made use of the + component of (B.4) in order to evaluate the covariant derivative in the above expression. In addition we have made use of the Bianchi identities (2.11) and the field equations (2.12)-(2.17).

\section{B.2.3 The (B.1) condition}

In order to show that (B.1) is implied from the independent KSEs we can compute the following,

$$
\begin{gathered}
\left(-\frac{1}{4} \tilde{R}-\Gamma^{i j} \tilde{\nabla}_{i} \tilde{\nabla}_{j}\right) \phi_{+}-\Gamma^{i} \tilde{\nabla}_{i}\left(\mathcal{A}_{1}\right) \\
+\left(\tilde{\nabla}_{i} \Phi \Gamma^{i}+\frac{1}{4} h_{i} \Gamma^{i}+\frac{1}{16} e^{\Phi} X_{l_{1} l_{2}} \Gamma^{l_{1} l_{2}}-\frac{1}{192} e^{\Phi} \tilde{G}_{l_{1} l_{2} l_{3} l_{4}} \Gamma^{l_{1} l_{2} l_{3} l_{4}}\right. \\
\left.+\Gamma_{11}\left(-\frac{1}{4} L_{l} \Gamma^{l}-\frac{1}{24} \tilde{H}_{l_{1} l_{2} l_{3}} \Gamma^{l_{1} l_{2} l_{3}}-\frac{1}{8} e^{\Phi} S+\frac{1}{16} e^{\Phi} \tilde{F}_{l_{1} l_{2}} \Gamma^{l_{1} l_{2}}\right)\right) \mathcal{A}_{1}=0
\end{gathered}
$$


where

$$
\begin{aligned}
\tilde{R}= & -2 \Delta-2 h^{i} \tilde{\nabla}_{i} \Phi-2 \tilde{\nabla}^{2} \Phi-\frac{1}{2} h^{2}+\frac{1}{2} L^{2}+\frac{1}{4} \tilde{H}^{2}+\frac{5}{2} e^{2 \Phi} S^{2} \\
& -\frac{1}{4} e^{2 \Phi} \tilde{F}^{2}+\frac{3}{4} e^{2 \Phi} X^{2}+\frac{1}{48} e^{2 \Phi} \tilde{G}^{2}
\end{aligned}
$$

and where we use the + component of (B.4) to evaluate the covariant derivative terms. In order to obtain (B.1) from these expressions we make use of the Bianchi identities (2.11), the field equations $(2.12)-(2.17)$, in particular in order to eliminate the $(\tilde{\nabla} \Phi)^{2}$ term. We have also made use of the +- component of the Einstein equation (2.18) in order to rewrite the scalar curvature $\tilde{R}$ in terms of $\Delta$. Therefore (B.1) follows from (B.4) and (B.7) together with the field equations and Bianchi identities mentioned above.

\section{B.2.4 The + (B.7) condition linear in $u$}

Since $\phi_{+}=\eta_{+}+u \Gamma_{+} \Theta_{-} \eta_{-}$, we must consider the part of the + component of (B.7) which is linear in $u$. On defining

$$
\begin{aligned}
\mathcal{B}_{1}= & \partial_{i} \Phi \Gamma^{i} \eta_{-}-\frac{1}{12} \Gamma_{11}\left(6 L_{i} \Gamma^{i}+\tilde{H}_{i j k} \Gamma^{i j k}\right) \eta_{-}+\frac{3}{8} e^{\Phi} \Gamma_{11}\left(2 S+\tilde{F}_{i j} \Gamma^{i j}\right) \eta_{-} \\
& +\frac{1}{4 \cdot 4 !} e^{\Phi}\left(12 X_{i j} \Gamma^{i j}+\tilde{G}_{j_{1} j_{2} j_{3} j_{4}} \Gamma^{j_{1} j_{2} j_{3} j_{4}}\right) \eta_{-}
\end{aligned}
$$

one finds that the $u$-dependent part of (B.7) is proportional to

$$
-\frac{1}{2} \Gamma^{i} \tilde{\nabla}_{i}\left(\mathcal{B}_{1}\right)+\Psi_{2} \mathcal{B}_{1}
$$

where

$$
\begin{aligned}
\Psi_{2}= & \tilde{\nabla}_{i} \Phi \Gamma^{i}+\frac{1}{8} h_{i} \Gamma^{i}-\frac{1}{16} e^{\Phi} X_{l_{1} l_{2}} \Gamma^{l_{1} l_{2}}-\frac{1}{192} e^{\Phi} \tilde{G}_{l_{1} l_{2} l_{3} l_{4}} \Gamma^{l_{1} l_{2} l_{3} l_{4}} \\
& +\Gamma_{11}\left(\frac{1}{48} \tilde{H}_{l_{1} l_{2} l_{3}} \Gamma^{l_{1} l_{2} l_{3}}+\frac{1}{8} L_{i} \Gamma^{i}+\frac{1}{16} e^{\Phi} \tilde{F}_{l_{1} l_{2}} \Gamma^{l_{1} l_{2}}+\frac{1}{8} e^{\Phi} S\right)
\end{aligned}
$$

We have made use of the - component of (B.4) in order to evaluate the covariant derivative in the above expression. In addition we have made use of the Bianchi identities (2.11) and the field equations (2.12)-(2.17).

\section{B.2.5 The (B.2) condition}

In order to show that (B.2) is implied from the independent KSEs we will show that it follows from (B.1). First act on (B.1) with the Dirac operator $\Gamma^{i} \tilde{\nabla}_{i}$ and use the field equations (2.12)-(2.17) and the Bianchi identities to eliminate the terms which contain derivatives of the fluxes and then use (B.1) to rewrite the $d h$-terms in terms of $\Delta$. Then use the conditions (B.4) and (B.5) to eliminate the $\partial_{i} \phi$-terms from the resulting expression, some of the remaining terms will vanish as a consequence of (B.1). After performing these calculations, the condition (B.2) is obtained, therefore it follows from section B.2.3 above that (B.2) is implied by (B.4) and (B.7) together with the field equations and Bianchi identities mentioned above. 


\section{B.2.6 The (B.3) condition}

In order to show that (B.3) is implied by the independent KSEs we can compute the following,

$$
\begin{gathered}
\left(\frac{1}{4} \tilde{R}+\Gamma^{i j} \tilde{\nabla}_{i} \tilde{\nabla}_{j}\right) \eta_{-}+\Gamma^{i} \tilde{\nabla}_{i}\left(\mathcal{B}_{1}\right) \\
+\left(-\tilde{\nabla}_{i} \Phi \Gamma^{i}+\frac{1}{4} h_{i} \Gamma^{i}+\frac{1}{16} e^{\Phi} X_{l_{1} l_{2}} \Gamma^{l_{1} l_{2}}+\frac{1}{192} e^{\Phi} \tilde{G}_{l_{1} l_{2} l_{3} l_{4}} \Gamma^{l_{1} l_{2} l_{3} l_{4}}\right. \\
\left.+\Gamma_{11}\left(-\frac{1}{4} L_{l} \Gamma^{l}+\frac{1}{24} \tilde{H}_{l_{1} l_{2} l_{3}} \Gamma^{l_{1} l_{2} l_{3}}-\frac{1}{8} e^{\Phi} S-\frac{1}{16} e^{\Phi} \tilde{F}_{l_{1} l_{2}} \Gamma^{l_{1} l_{2}}\right)\right) \mathcal{B}_{1}=0,
\end{gathered}
$$

where we use the - component of (B.4) to evaluate the covariant derivative terms. The expression above vanishes identically since the - component of (B.7) is equivalent to $\mathcal{B}_{1}=0$. In order to obtain (B.3) from these expressions we make use of the Bianchi identities (2.11) and the field equations (2.12)-(2.17). Therefore (B.3) follows from (B.4) and (B.7) together with the field equations and Bianchi identities mentioned above.

\section{B.2.7 The + (B.4) condition linear in $u$}

Next consider the part of the + component of (B.4) which is linear in $u$. First compute

$$
\left(\Gamma^{j}\left(\tilde{\nabla}_{j} \tilde{\nabla}_{i}-\tilde{\nabla}_{i} \tilde{\nabla}_{j}\right)-\frac{1}{2} \tilde{R}_{i j} \Gamma^{j}\right) \eta_{-}-\tilde{\nabla}_{i}\left(\mathcal{B}_{1}\right)-\Psi_{i} \mathcal{B}_{1}=0
$$

where

$$
\Psi_{i}=\frac{1}{4} h_{i}-\Gamma_{11}\left(\frac{1}{4} L_{i}+\frac{1}{8} \tilde{H}_{i j k} \Gamma^{j k}\right)
$$

and where we have made use of the - component of (B.4) to evaluate the covariant derivative terms. The resulting expression corresponds to the expression obtained by expanding out the $u$-dependent part of the + component of (B.4) by using the - component of (B.4) to evaluate the covariant derivative. We have made use of the Bianchi identities (2.11) and the field equations (2.12)-(2.16).

\section{Calculation of Laplacian of $\left\|\eta_{ \pm}\right\|^{2}$}

In this appendix, we calculate the Laplacian of $\left\|\eta_{ \pm}\right\|^{2}$, which will be particularly useful in the analysis of the global properties of IIA horizons in section 3. We shall consider the modified gravitino KSE (3.3) defined in section 3.1, and we shall assume throughout that the modified Dirac equation $\mathscr{D}^{( \pm)} \eta_{ \pm}=0$ holds, where $\mathscr{D}^{( \pm)}$is defined in (3.5). Also, $\Psi_{i}^{( \pm)}$ and $\mathcal{A}^{( \pm)}$are defined by (2.28) and (2.29), and $\Psi^{( \pm)}$is defined by (3.2).

To proceed, we compute the Laplacian

$$
\tilde{\nabla}^{i} \tilde{\nabla}_{i}\left\|\eta_{ \pm}\right\|^{2}=2\left\langle\eta_{ \pm}, \tilde{\nabla}^{i} \tilde{\nabla}_{i} \eta_{ \pm}\right\rangle+2\left\langle\tilde{\nabla}^{i} \eta_{ \pm}, \tilde{\nabla}_{i} \eta_{ \pm}\right\rangle
$$


To evaluate this expression note that

$$
\begin{aligned}
\tilde{\nabla}^{i} \tilde{\nabla}_{i} \eta_{ \pm} & =\Gamma^{i} \tilde{\nabla}_{i}\left(\Gamma^{j} \tilde{\nabla}_{j} \eta_{ \pm}\right)-\Gamma^{i j} \tilde{\nabla}_{i} \tilde{\nabla}_{j} \eta_{ \pm} \\
& =\Gamma^{i} \tilde{\nabla}_{i}\left(\Gamma^{j} \tilde{\nabla}_{j} \eta_{ \pm}\right)+\frac{1}{4} \tilde{R} \eta_{ \pm} \\
& =\Gamma^{i} \tilde{\nabla}_{i}\left(-\Psi^{( \pm)} \eta_{ \pm}-q \mathcal{A}^{( \pm)} \eta_{ \pm}\right)+\frac{1}{4} \tilde{R} \eta_{ \pm}
\end{aligned}
$$

It follows that

$$
\begin{aligned}
\left\langle\eta_{ \pm}, \tilde{\nabla}^{i} \tilde{\nabla}_{i} \eta_{ \pm}\right\rangle= & \frac{1}{4} \tilde{R}\left\|\eta_{ \pm}\right\|^{2}+\left\langle\eta_{ \pm}, \Gamma^{i} \tilde{\nabla}_{i}\left(-\Psi^{( \pm)}-q \mathcal{A}^{( \pm)}\right) \eta_{ \pm}\right\rangle \\
& +\left\langle\eta_{ \pm}, \Gamma^{i}\left(-\Psi^{( \pm)}-q \mathcal{A}^{( \pm)}\right) \tilde{\nabla}_{i} \eta_{ \pm}\right\rangle
\end{aligned}
$$

and also

$$
\begin{aligned}
\left\langle\tilde{\nabla}^{i} \eta_{ \pm}, \tilde{\nabla}_{i} \eta_{ \pm}\right\rangle= & \left\langle\hat{\nabla}^{( \pm) i} \eta_{ \pm}, \hat{\nabla}_{i}^{( \pm)} \eta_{ \pm}\right\rangle-2\left\langle\eta_{ \pm},\left(\Psi^{( \pm) i}+\kappa \Gamma^{i} \mathcal{A}^{( \pm)}\right)^{\dagger} \tilde{\nabla}_{i} \eta_{ \pm}\right\rangle \\
& -\left\langle\eta_{ \pm},\left(\Psi^{( \pm) i}+\kappa \Gamma^{i} \mathcal{A}^{( \pm)}\right)^{\dagger}\left(\Psi_{i}^{( \pm)}+\kappa \Gamma_{i} \mathcal{A}^{( \pm)}\right) \eta_{ \pm}\right\rangle \\
= & \left\|\hat{\nabla}^{( \pm)} \eta_{ \pm}\right\|^{2}-2\left\langle\eta_{ \pm}, \Psi^{( \pm) i \dagger} \tilde{\nabla}_{i} \eta_{ \pm}\right\rangle-2 \kappa\left\langle\eta_{ \pm}, \mathcal{A}^{( \pm) \dagger} \Gamma^{i} \tilde{\nabla}_{i} \eta_{ \pm}\right\rangle \\
& -\left\langle\eta_{ \pm},\left(\Psi^{( \pm) i \dagger} \Psi_{i}^{( \pm)}+2 \kappa \mathcal{A}^{( \pm) \dagger} \Psi^{( \pm)}+8 \kappa^{2} \mathcal{A}^{( \pm) \dagger} \mathcal{A}^{( \pm)}\right) \eta_{ \pm}\right\rangle \\
= & \left\|\hat{\nabla}^{( \pm)} \eta_{ \pm}\right\|^{2}-2\left\langle\eta_{ \pm}, \Psi^{( \pm) i \dagger} \tilde{\nabla}_{i} \eta_{ \pm}\right\rangle-\left\langle\eta_{ \pm}, \Psi^{( \pm) i \dagger} \Psi_{i}^{( \pm)} \eta_{ \pm}\right\rangle \\
& +\left(2 \kappa q-8 \kappa^{2}\right)\left\|\mathcal{A}^{( \pm)} \eta_{ \pm}\right\|^{2} .
\end{aligned}
$$

Therefore,

$$
\begin{aligned}
\frac{1}{2} \tilde{\nabla}^{i} \tilde{\nabla}_{i}\left\|\eta_{ \pm}\right\|^{2}= & \left\|\hat{\nabla}^{( \pm)} \eta_{ \pm}\right\|^{2}+\left(2 \kappa q-8 \kappa^{2}\right)\left\|\mathcal{A}^{( \pm)} \eta_{ \pm}\right\|^{2} \\
& +\left\langle\eta_{ \pm},\left(\frac{1}{4} \tilde{R}+\Gamma^{i} \tilde{\nabla}_{i}\left(-\Psi^{( \pm)}-q \mathcal{A}^{( \pm)}\right)-\Psi^{( \pm) i \dagger} \Psi_{i}^{( \pm)}\right) \eta_{ \pm}\right\rangle \\
& +\left\langle\eta_{ \pm},\left(\Gamma^{i}\left(-\Psi^{( \pm)}-q \mathcal{A}^{( \pm)}\right)-2 \Psi^{( \pm) i \dagger}\right) \tilde{\nabla}_{i} \eta_{ \pm}\right\rangle
\end{aligned}
$$

In order to simplify the expression for the Laplacian, we shall attempt to rewrite the third line in (C.5) as

$$
\left\langle\eta_{ \pm},\left(\Gamma^{i}\left(-\Psi^{( \pm)}-q \mathcal{A}^{( \pm)}\right)-2 \Psi^{( \pm) i \dagger}\right) \tilde{\nabla}_{i} \eta_{ \pm}\right\rangle=\left\langle\eta_{ \pm}, \mathcal{F}^{( \pm)} \Gamma^{i} \tilde{\nabla}_{i} \eta_{ \pm}\right\rangle+W^{( \pm) i} \tilde{\nabla}_{i}\left\|\eta_{ \pm}\right\|^{2}
$$

where $\mathcal{F}^{( \pm)}$is linear in the fields and $W^{( \pm) i}$ is a vector. This expression is particularly advantageous, because the first term on the r.h.s. can be rewritten using the horizon Dirac equation, and the second term is consistent with the application of the maximum principle/integration by parts arguments which are required for the generalized Lichnerowicz 
theorems. In order to rewrite (C.6) in this fashion, note that

$$
\begin{aligned}
\Gamma^{i}\left(\Psi^{( \pm)}+q \mathcal{A}^{( \pm)}\right)+2 \Psi^{( \pm) i \dagger}= & \left(\mp h^{i} \mp(q+1) \Gamma_{11} L^{i}+\frac{1}{2}(q+1) \Gamma_{11} \tilde{H}^{i} \ell_{1} \ell_{2} \Gamma^{\ell_{1} \ell_{2}}+2 q \tilde{\nabla}^{i} \Phi\right) \\
& +\left( \pm \frac{1}{4} h_{j} \Gamma^{j} \pm\left(\frac{q}{2}+\frac{1}{4}\right) \Gamma_{11} L_{j} \Gamma^{j}\right. \\
& \left.-\left(\frac{q}{12}+\frac{1}{8}\right) \Gamma_{11} \tilde{H}_{\ell_{1} \ell_{2} \ell_{3}} \Gamma^{\ell_{1} \ell_{2} \ell_{3}}-q \tilde{\nabla}_{j} \Phi \Gamma^{j}\right) \Gamma^{i} \\
& \mp \frac{1}{8}(q+1) e^{\Phi} X_{\ell_{1} \ell_{2}} \Gamma^{i} \Gamma^{\ell_{1} \ell_{2}}+\frac{1}{96}(q+1) e^{\Phi} \tilde{G}_{\ell_{1} \ell_{2} \ell_{3} \ell_{4}} \Gamma^{i} \Gamma^{\ell_{1} \ell_{2} \ell_{3} \ell_{4}} \\
& +(q+1) \Gamma_{11}\left( \pm \frac{3}{4} e^{\Phi} S \Gamma^{i}-\frac{3}{8} e^{\Phi} \tilde{F}_{\ell_{1} \ell_{2}} \Gamma^{i} \Gamma^{\ell_{1} \ell_{2}}\right) .
\end{aligned}
$$

One finds that (C.6) is only possible for $q=-1$ and thus we have

$$
\begin{aligned}
W^{( \pm) i} & =\frac{1}{2}\left(2 \tilde{\nabla}^{i} \Phi \pm h^{i}\right) \\
\mathcal{F}^{( \pm)} & =\mp \frac{1}{4} h_{j} \Gamma^{j}-\tilde{\nabla}_{j} \Phi \Gamma^{j}+\Gamma_{11}\left( \pm \frac{1}{4} L_{j} \Gamma^{j}+\frac{1}{24} \tilde{H}_{\ell_{1} \ell_{2} \ell_{3}} \Gamma^{\ell_{1} \ell_{2} \ell_{3}}\right) .
\end{aligned}
$$

We remark that $\dagger$ is the adjoint with respect to the Spin(8)-invariant inner product $\langle$,$\rangle . In order to compute the adjoints above we note that the Spin(8)-invariant inner$ product restricted to the Majorana representation is positive definite and real, and so symmetric. With respect to this the gamma matrices are Hermitian and thus the skew symmetric products $\Gamma^{[k]}$ of $k \operatorname{Spin}(8)$ gamma matrices are Hermitian for $k=0(\bmod 4)$ and $k=1(\bmod 4)$ while they are anti-Hermitian for $k=2(\bmod 4)$ and $k=3(\bmod 4)$. The $\Gamma_{11}$ matrix is also Hermitian since it is a product of the first 10 gamma matrices and we take $\Gamma_{0}$ to be anti-Hermitian. It also follows that $\Gamma_{11} \Gamma^{[k]}$ is Hermitian for $k=0(\bmod 4)$ and $k=3(\bmod 4)$ and anti-Hermitian for $k=1(\bmod 4)$ and $k=2(\bmod 4)$. This also implies the following identities

$$
\left\langle\eta_{+}, \Gamma^{[k]} \eta_{+}\right\rangle=0, \quad k=2(\bmod 4) \text { and } k=3(\bmod 4)
$$

and

$$
\left\langle\eta_{+}, \Gamma_{11} \Gamma^{[k]} \eta_{+}\right\rangle=0, \quad k=1(\bmod 4) \text { and } k=2(\bmod 4) .
$$

It follows that

$$
\begin{aligned}
\frac{1}{2} \tilde{\nabla}^{i} \tilde{\nabla}_{i}\left\|\eta_{ \pm}\right\|^{2}= & \left\|\hat{\nabla}^{( \pm)} \eta_{ \pm}\right\|^{2}+\left(-2 \kappa-8 \kappa^{2}\right)\left\|\mathcal{A}^{( \pm)} \eta_{ \pm}\right\|^{2}+W^{( \pm) i} \tilde{\nabla}_{i}\left\|\eta_{ \pm}\right\|^{2} \\
& +\left\langle\eta_{ \pm},\left(\frac{1}{4} \tilde{R}+\Gamma^{i} \tilde{\nabla}_{i}\left(-\Psi^{( \pm)}+\mathcal{A}^{( \pm)}\right)\right.\right. \\
& \left.\left.-\Psi^{( \pm) i \dagger} \Psi_{i}^{( \pm)}+\mathcal{F}^{( \pm)}\left(-\Psi^{( \pm)}+\mathcal{A}^{( \pm)}\right)\right) \eta_{ \pm}\right\rangle
\end{aligned}
$$

It is also useful to evaluate $\tilde{R}$ using (2.19) and the dilaton field equation (2.17); we obtain

$$
\begin{aligned}
\tilde{R}= & -\tilde{\nabla}^{i}\left(h_{i}\right)+\frac{1}{2} h^{2}-4(\tilde{\nabla} \Phi)^{2}-2 h^{i} \tilde{\nabla}_{i} \Phi-\frac{3}{2} L^{2}+\frac{5}{12} \tilde{H}^{2} \\
& +\frac{7}{2} e^{2 \Phi} S^{2}-\frac{5}{4} e^{2 \Phi} \tilde{F}^{2}+\frac{3}{4} e^{2 \Phi} X^{2}-\frac{1}{48} e^{2 \Phi} \tilde{G}^{2} .
\end{aligned}
$$


One obtains, upon using the field equations and Bianchi identities,

$$
\begin{aligned}
\left(\frac{1}{4} \tilde{R}+\right. & \left.\Gamma^{i} \tilde{\nabla}_{i}\left(-\Psi^{( \pm)}+\mathcal{A}^{( \pm)}\right)-\Psi^{( \pm) i \dagger} \Psi_{i}^{( \pm)}+\mathcal{F}^{( \pm)}\left(-\Psi^{( \pm)}+\mathcal{A}^{( \pm)}\right)\right) \eta_{ \pm} \\
= & {\left[\left( \pm \frac{1}{4} \tilde{\nabla}_{\ell_{1}}\left(h_{\ell_{2}}\right) \mp \frac{1}{16} \tilde{H}_{\ell_{1} \ell_{2}}^{i} L_{i}\right) \Gamma^{\ell_{1} \ell_{2}}+\left( \pm \frac{1}{8} \tilde{\nabla}_{\ell_{1}}\left(e^{\Phi} X_{\ell_{2} \ell_{3}}\right)+\frac{1}{24} \tilde{\nabla}^{i}\left(e^{\Phi} \tilde{G}_{i \ell_{1} \ell_{2} \ell_{3}}\right)\right.\right.} \\
& \mp \frac{1}{96} e^{\Phi} h^{i} \tilde{G}_{i \ell_{1} \ell_{2} \ell_{3}}-\frac{1}{32} e^{\Phi} X_{\ell_{1} \ell_{2}} h_{\ell_{3}} \mp \frac{1}{8} e^{\Phi} \tilde{\nabla}_{\ell_{1}} \Phi X_{\ell_{2} \ell_{3}}-\frac{1}{24} e^{\Phi} \tilde{\nabla}^{i} \Phi \tilde{G}_{i \ell_{1} \ell_{2} \ell_{3}} \\
& \left.\mp \frac{1}{32} e^{\Phi} \tilde{F}_{\ell_{1} \ell_{2}} L_{\ell_{3}} \mp \frac{1}{96} e^{\Phi} S \tilde{H}_{\ell_{1} \ell_{2} \ell_{3}}-\frac{1}{32} e^{\Phi} \tilde{F}_{\ell_{1}}^{i} \tilde{H}_{i \ell_{2} \ell_{3}}\right) \Gamma^{\ell_{1} \ell_{2} \ell_{3}} \\
& +\Gamma_{11}\left(\left(\mp \frac{1}{4} \tilde{\nabla}_{\ell}\left(e^{\Phi} S\right)-\frac{1}{4} \tilde{\nabla}^{i}\left(e^{\Phi} \tilde{F}_{i \ell}\right)+\frac{1}{16} e^{\Phi} S h_{\ell} \pm \frac{1}{16} e^{\Phi} h^{i} \tilde{F}_{i \ell} \pm \frac{1}{4} e^{\Phi} \tilde{\nabla}_{\ell} \Phi S\right.\right. \\
& \left.+\frac{1}{4} e^{\Phi} \tilde{\nabla}^{i} \Phi \tilde{F}_{i \ell}+\frac{1}{16} e^{\Phi} L^{i} X_{i \ell} \mp \frac{1}{32} e^{\Phi} \tilde{H}^{i j} X_{\ell} X_{i j}-\frac{1}{96} e^{\Phi} \tilde{G}^{i j k} \tilde{H}_{i j k}\right) \Gamma^{\ell} \\
& +\left(\mp \frac{1}{4} \tilde{\nabla}_{\ell_{1}}\left(L_{\ell_{2}}\right)-\frac{1}{8} \tilde{\nabla}^{i}\left(\tilde{H}_{i \ell_{1} \ell_{2}}\right)+\frac{1}{4} \tilde{\nabla}^{i} \Phi \tilde{H}_{i \ell_{1} \ell_{2}} \pm \frac{1}{16} h^{i} \tilde{H}_{i \ell_{1} \ell_{2}}\right) \Gamma^{\ell_{1} \ell_{2}} \\
& \left.\left.+\left( \pm \frac{1}{384} e^{\Phi} \tilde{G}_{\ell_{1} \ell_{2} \ell_{3} \ell_{4}} L_{\ell_{5}} \pm \frac{1}{192} e^{\Phi} \tilde{H}_{\ell_{1} \ell_{2} \ell_{3}} X_{\ell_{4} \ell_{5}}+\frac{1}{192} e^{\Phi} \tilde{G}_{\ell_{1} \ell_{2} \ell_{3}}^{i} \tilde{H}_{i \ell_{4} \ell_{5}}\right) \Gamma^{\ell_{1} \ell_{2} \ell_{3} \ell_{4} \ell_{5}}\right)\right] \eta_{ \pm} \\
& +\frac{1}{2}(1 \mp 1)\left(h^{i} \tilde{\nabla}_{i} \Phi-\frac{1}{2} \tilde{\nabla}^{i} h_{i}\right) \eta_{ \pm} .
\end{aligned}
$$

Note that with the exception of the final line of the r.h.s. of (C.14), all terms on the r.h.s. of the above expression give no contribution to the second line of (C.12), using (C.10) and (C.11), since all these terms in (C.14) are anti-Hermitian and thus the bilinears vanish. Furthermore, the contribution to the Laplacian of $\left\|\eta_{+}\right\|^{2}$ from the final line of (C.14) also vanishes; however the final line of (C.14) does give a contribution to the second line of (C.12) in the case of the Laplacian of $\left\|\eta_{-}\right\|^{2}$. We proceed to consider the Laplacians of $\left\|\eta_{ \pm}\right\|^{2}$ separately, as the analysis of the conditions imposed by the global properties of $\mathcal{S}$ differs slightly in the two cases.

For the Laplacian of $\left\|\eta_{+}\right\|^{2}$, we obtain from (C.12):

$$
\tilde{\nabla}^{i} \tilde{\nabla}_{i}\left\|\eta_{+}\right\|^{2}-\left(2 \tilde{\nabla}^{i} \Phi+h^{i}\right) \tilde{\nabla}_{i}\left\|\eta_{+}\right\|^{2}=2\left\|\hat{\nabla}^{(+)} \eta_{+}\right\|^{2}-\left(4 \kappa+16 \kappa^{2}\right)\left\|\mathcal{A}^{(+)} \eta_{+}\right\|^{2} .
$$

This proves (3.6).

The Laplacian of $\left\|\eta_{-}\right\|^{2}$ is calculated from (C.12), on taking account of the contribution to the second line of (C.12) from the final line of (C.14). One obtains

$$
\tilde{\nabla}^{i}\left(e^{-2 \Phi} V_{i}\right)=-2 e^{-2 \Phi}\left\|\hat{\nabla}^{(-)} \eta_{-}\right\|^{2}+e^{-2 \Phi}\left(4 \kappa+16 \kappa^{2}\right)\left\|\mathcal{A}^{(-)} \eta_{-}\right\|^{2},
$$

where

$$
V=-d\left\|\eta_{-}\right\|^{2}-\left\|\eta_{-}\right\|^{2} h
$$

This proves (3.9) and completes the proof.

Open Access. This article is distributed under the terms of the Creative Commons Attribution License (CC-BY 4.0), which permits any use, distribution and reproduction in any medium, provided the original author(s) and source are credited. 


\section{References}

[1] U. Gran, J. Gutowski and G. Papadopoulos, Index theory and dynamical symmetry enhancement near IIB horizons, JHEP 11 (2013) 104 [arXiv: 1306.5765] [INSPIRE].

[2] J. Grover, J. Gutowski, G. Papadopoulos and W.A. Sabra, Index Theory and Supersymmetry of $5 D$ Horizons, JHEP 06 (2014) 020 [arXiv:1303.0853] [inSPIRE].

[3] J. Gutowski and G. Papadopoulos, Index theory and dynamical symmetry enhancement of M-horizons, JHEP 05 (2013) 088 [arXiv:1303.0869] [INSPIRE].

[4] B. Carter, Black Holes, C. de Witt and B.S. de Witt eds., Gordon and Breach, New York U.S.A (1973).

[5] G.W. Gibbons, Aspects of Supergravity Theories, in Supersymmetry, Supergravity and Related Topics, F. del Aguila, J.A. de Azc'arraga and L.E. Ibanez eds., World Scientific, Singapore (1985).

[6] G.W. Gibbons and P.K. Townsend, Vacuum interpolation in supergravity via super p-branes, Phys. Rev. Lett. 71 (1993) 3754 [hep-th/9307049] [INSPIRE].

[7] O. Aharony, S.S. Gubser, J.M. Maldacena, H. Ooguri and Y. Oz, Large-N field theories, string theory and gravity, Phys. Rept. 323 (2000) 183 [hep-th/9905111] [INSPIRE].

[8] V. Moncrief and J. Isenberg, Symmetries of cosmological Cauchy horizons, Commun. Math. Phys. 89 (1983) 387 [INSPIRE].

[9] H. Friedrich, I. Racz and R.M. Wald, On the rigidity theorem for space-times with a stationary event horizon or a compact Cauchy horizon, Commun. Math. Phys. 204 (1999) 691 [gr-qc/9811021] [INSPIRE].

[10] I. Bakas and K. Sfetsos, T duality and world sheet supersymmetry, Phys. Lett. B 349 (1995) 448 [hep-th/9502065] [INSPIRE].

[11] M.J. Duff, H. Lü and C.N. Pope, Supersymmetry without supersymmetry, Phys. Lett. B 409 (1997) 136 [hep-th/9704186] [INSPIRE].

[12] M. Huq and M.A. Namazie, Kaluza-Klein Supergravity in Ten-dimensions, Class. Quant. Grav. 2 (1985) 293 [Erratum ibid. 2 (1985) 597] [INSPIRE].

[13] F. Giani and M. Pernici, N=2 supergravity in ten-dimensions, Phys. Rev. D 30 (1984) 325 [INSPIRE].

[14] I.C.G. Campbell and P.C. West, $N=2 D=10$ Nonchiral Supergravity and Its Spontaneous Compactification, Nucl. Phys. B 243 (1984) 112 [inSPIRE].

[15] L.J. Romans, Massive N=2a Supergravity in Ten-Dimensions, Phys. Lett. B 169 (1986) 374 [INSPIRE].

[16] E.A. Bergshoeff, J. Hartong, P.S. Howe, T. Ortín and F. Riccioni, IIA/ IIB Supergravity and Ten-forms, JHEP 05 (2010) 061 [arXiv:1004.1348] [INSPIRE].

[17] M.F. Atiyah and I.M. Singer, The Index of elliptic operators. 1, Annals Math. 87 (1968) 484 [INSPIRE].

[18] J.M. Figueroa-O'Farrill and G. Papadopoulos, Maximally supersymmetric solutions of ten-dimensional and eleven-dimensional supergravities, JHEP 03 (2003) 048 [hep-th/0211089] [INSPIRE]. 
[19] U. Gran, G. Papadopoulos and C. von Schultz, Supersymmetric geometries of IIA supergravity I, JHEP 05 (2014) 024 [arXiv:1401.6900] [INSPIRE].

[20] J. Figueroa-O'Farrill and N. Hustler, The homogeneity theorem for supergravity backgrounds, JHEP 10 (2012) 014 [arXiv: 1208.0553] [INSPIRE].

[21] U. Gran, J. Gutowski, G. Papadopoulos and D. Roest, N=31 is not IIB, JHEP 02 (2007) 044 [hep-th/0606049] [INSPIRE].

[22] U. Gran, J. Gutowski, G. Papadopoulos and D. Roest, IIB solutions with $N>28$ Killing spinors are maximally supersymmetric, JHEP 12 (2007) 070 [arXiv:0710.1829] [INSPIRE].

[23] U. Gran, J. Gutowski and G. Papadopoulos, Classification of IIB backgrounds with 28 supersymmetries, JHEP 01 (2010) 044 [arXiv:0902.3642] [INSPIRE].

[24] I.A. Bandos, J.A. de Azcarraga and O. Varela, On the absence of BPS preonic solutions in IIA and IIB supergravities, JHEP 09 (2006) 009 [hep-th/0607060] [INSPIRE]. 